\title{
Convergence and stability of modified multi-step Noor iterative procedure with errors for strictly hemicontractive-type mappings in Banach spaces
}

\section{Md. Asaduzzaman ${ }^{1 *}$ (D)}

"Correspondence:

masad_iu_math@yahoo.com 'Department of Mathematics, Islamic University, Kushtia, 7003, Bangladesh

\section{Springer}

\begin{abstract}
In this paper, we introduce and study a modified multi-step Noor iterative procedure with errors for two Lipschitz strictly hemicontractive-type mappings in arbitrary Banach spaces and constitute its convergence and stability. The obtained results in this paper generalize and extend the corresponding result of Hussain et al. (Fixed Point Theory Appl. 2012:160, 2012) and some analogous results of several authors in the literature. Finally, a numerical example is included to illustrate our analytical results and to display the efficiency of our proposed novel iterative procedure with errors.
\end{abstract}

MSC: 47J25; 47H09; 47H10; 54H25

Keywords: Modified multi-step Noor iterative procedure with errors; Lipschitz strictly hemicontractive-type mapping; Convergence; Common stability; Almost common-stability

\section{Introduction}

In the last few decades, fixed-point theorem-based iterative procedures whose convergence established on the strictly hemicontractive-type mappings earn a great attention for its rigorous applications in the diverse fields of various mathematical problems; see for instance [2-5] and the references cited therein. Application of strictly hemicontractive-type mapping was initiated by Chidume and Osilike [4] for improving the consequence of Chidume [5]. After Chidume and Osilike [4], several researchers studied strictly hemicontractive-type mapping in many directions; see for instance [1-3, 6-21] and the references cited therein. Among the articles cited in [1-3, 6-21], Hussain et al. [1] studied Lipschitz strictly hemicontractive-type mapping in arbitrary Banach spaces to extend and improve the equivalent consequences of the monographs [4, 5, 12-15].

Throughout this paper, $\mathbb{R}$ denotes the set of real numbers, $B$ represents a nonempty subset of an arbitrary Banach space $X$ and $X^{*}$ is a dual space of $X$. Let $T$ be a single-valued map from $B$ into itself, then $r \in B$ is called a fixed point of $T$ iff $T(r)=r$. The symbols $D_{T}, R_{T}$ and $F_{T}$ denote the domain of $T$, the range of $T$ and the set of fixed points of $T$

(c) The Author(s) 2021. This article is licensed under a Creative Commons Attribution 4.0 International License, which permits use, sharing, adaptation, distribution and reproduction in any medium or format, as long as you give appropriate credit to the original author(s) and the source, provide a link to the Creative Commons licence, and indicate if changes were made. The images or other third party material in this article are included in the article's Creative Commons licence, unless indicated otherwise in a credit line to the material. If material is not included in the article's Creative Commons licence and your intended use is not permitted by statutory regulation or exceeds the permitted use, you will need to obtain permission directly from the copyright holder. To view a copy of this licence, visit http://creativecommons.org/licenses/by/4.0/. 
respectively. Let $J: X \rightarrow 2^{X^{*}}$ be a normalized duality mapping given by

$$
J(r)=\left\{g^{*} \in X^{*}:\left\langle r, g^{*}\right\rangle=\|r\|^{2}=\left\|g^{*}\right\|^{2}\right\} .
$$

The mapping $T$ is called Lipschitzian if there exists a $L>0$ such that

$$
\|T q-T r\| \leq L\|q-r\|
$$

for all $q, r \in B$. If $L=1$, then $T$ is called a non-expansive mapping, and if $0 \leq L<1$, then $T$ is called a contraction mapping.

The mapping $T$ is called a strictly hemicontractive mapping if $F_{T} \neq \varphi$ and if there exists a constant $t>1$ such that

$$
\|q-r\| \leq\left\|\left(1+t^{\prime}\right)(q-r)-t^{\prime} t(T q-T r)\right\|
$$

for all $q \in D_{T}, r \in F_{T}$ and $t^{\prime}>0$.

If the mapping $T$ satisfies both inequalities (1.1) and (1.2), then it is called a Lipschitz strictly hemicontractive mapping.

The mapping $T$ is called asymptotically non-expansive on $B$ if there exists a sequence $\left\{s_{n}\right\}$ in $[0, \infty)$ with $\lim _{n \rightarrow \infty} s_{n}=0$ such that, for each $p, q \in B$,

$$
\left\|T^{n} p-T^{n} q\right\| \leq\left(1+s_{n}\right)\|p-q\|, \quad \forall n \geq 1 .
$$

$T$ is called an asymptotically non-expansive mapping in the intermediate sense if $T$ is uniformly continuous and

$$
\lim \sup _{n \rightarrow \infty} \sup _{p, q \in B}\left(\left\|T^{n} p-T^{n} q\right\|-\|p-q\|\right) \leq 0 .
$$

The mapping $T$ is called an asymptotically quasi-non-expansive mapping if there exists a sequence $\left\{s_{n}\right\}$ in $[0, \infty)$ with $\lim _{n \rightarrow \infty} s_{n}=0$ such that, for all $p \in B, q \in F_{T}$,

$$
\left\|T^{n} p-q\right\| \leq\left(1+s_{n}\right)\|p-q\|, \quad \forall n \geq 1 .
$$

According to the definitions, it is clear that an asymptotically non-expansive mapping must be an asymptotically non-expansive mapping in the intermediate sense and an asymptotically quasi-non-expansive mapping, but the converse is not always true. We may justify this concept by using the following example.

Example 1.1 (See [22]) Let $X=\mathbb{R}$ (with the usual norm), $B=\left[-\frac{1}{\pi}, \frac{1}{\pi}\right]$ and $|t|<1$. For each $u \in B$, we define

$$
T u= \begin{cases}t u \sin \frac{1}{u} & \text { if } u \neq 0, \\ 0 & \text { if } u=0 .\end{cases}
$$

Then $T$ is an asymptotically non-expansive mapping in the intermediate sense and an asymptotically quasi-non-expansive mapping, but is not a Lipschitzian mapping, thus it is not an asymptotically non-expansive mapping as well as it is not a Lipschitz strictly hemicontractive mapping. 
Remark 1.2 We note that an asymptotically non-expansive mapping in the intermediate sense or an asymptotically quasi-non-expansive mapping is not always a Lipschitz strictly hemicontractive mapping.

We now provide an example which shows that a Lipschitz strictly hemicontractive mapping is also an asymptotically non-expansive mapping.

Example 1.3 Let $X=\mathbb{R}$ with the usual norm and $B=[0,2 \pi]$. Define $T: B \rightarrow B$ by $T u=$ $\frac{u \cos u}{2}$ for each $u \in B$. Clearly $F_{T}=\{0\}$. For each $u \in D_{T}, r \in F_{T}, t^{\prime}>0$, choose $t=2$. Then we have

$$
\begin{aligned}
\left|\left(1+t^{\prime}\right)(u-r)-t^{\prime} t(T u-T r)\right| & =\left|\left(1+t^{\prime}\right) u-2 t^{\prime} T u\right| \\
& =\left|\left(1+t^{\prime}\right) u-2 t^{\prime} \cdot \frac{u \cos u}{2}\right| \\
& \geq\left(1+t^{\prime}\right) u-t^{\prime} u=u=|u-r|
\end{aligned}
$$

and hence $T$ is a strictly hemicontractive mapping.

And, if we consider $u=\pi, v=2 \pi$, then it is easy to see that $|u-v|=\pi$ and hence

$$
\begin{aligned}
|T u-T v| & =\left|\frac{u \cos u}{2}-\frac{v \cos v}{2}\right|=\frac{\pi}{2}|\cos \pi-2 \cos 2 \pi| \\
& <\frac{\pi}{2}|\cos \pi-\cos 2 \pi| \\
& \leq \frac{\pi}{2}|\pi-2 \pi|=\frac{\pi^{2}}{2}=L|u-v|,
\end{aligned}
$$

for all $u, v \in B$ and $L=\frac{\pi}{2}>0$. Thus, $T$ is a Lipschitz strictly hemicontractive mapping.

Furthermore, for a sequence $\left\{\frac{1}{n}\right\}$ we have

$$
\begin{aligned}
\left|T^{n} u-T^{n} v\right| & =\frac{1}{2^{n}}|u \cos u-v \cos v|=\frac{\pi}{2^{n}}|\cos \pi-2 \cos 2 \pi| \\
& <\frac{\pi}{2^{n}}|\cos \pi-\cos 2 \pi| \\
& \leq \frac{\pi}{2^{n}}|\pi-2 \pi|=\frac{\pi}{2^{n}} \cdot \pi<\left(1+\frac{1}{n}\right) \cdot \pi=\left(1+\frac{1}{n}\right)|u-v|,
\end{aligned}
$$

for all $u, v \in B$ and $n \geq 1$. Hence, $T$ is an asymptotically non-expansive mapping. Therefore, a Lipschitz strictly hemicontractive mapping may also be an asymptotically nonexpansive mapping.

The following example shows that a strictly hemicontractive mapping is neither a Lipschitzian mapping nor an asymptotically non-expansive mapping.

Example 1.4 (See [23]) Let $X=\mathbb{R}$ (with the usual norm), $B=[0,1]$ and let $\varphi$ be the Cantor ternary function. If we define $T: B \rightarrow X$ by

$$
T u= \begin{cases}\frac{u}{2} & \text { if } 0 \leq u \leq \frac{1}{2}, \\ \varphi((1-u) / 2) & \text { if } \frac{1}{2}<u \leq 1\end{cases}
$$


then $T^{n} u \rightarrow 0$ uniformly on $B$ and $T$ is a strictly hemicontractive mapping. But we observe that $T$ is neither a Lipschitzian mapping nor an asymptotically non-expansive mapping.

In 2006, Plubtieng and Wangkeeree [24] introduced and studied the following multistep Noor iterative procedure with errors for some special type of asymptotically nonexpansive mappings (asymptotically non-expansive mapping in the intermediate sense and asymptotically quasi-non-expansive mapping) in Banach spaces: For a given $u_{1} \in B$, and a fixed $m \in \mathbf{N}$ (set of all positive integers), the iterative sequences $\left\{u_{n}^{(1)}\right\},\left\{u_{n}^{(2)}\right\}, \ldots,\left\{u_{n}^{(m)}\right\}$ defined by

$$
\left.\begin{array}{l}
u_{n}^{(1)}=a_{n}^{(1)} T^{n} u_{n}+b_{n}^{(1)} u_{n}+c_{n}^{(1)} v_{n}^{(1)}, \\
u_{n}^{(2)}=a_{n}^{(2)} T^{n} u_{n}^{(1)}+b_{n}^{(2)} u_{n}+c_{n}^{(2)} v_{n}^{(2)}, \\
\cdots \cdots \\
u_{n}^{(m-1)}=a_{n}^{(m-1)} T^{n} u_{n}^{(m-2)}+b_{n}^{(m-1)} u_{n}+c_{n}^{(m-1)} v_{n}^{(m-1)}, \\
u_{n+1}=u_{n}^{(m)}=a_{n}^{(m)} T^{n} u_{n}^{(m-1)}+b_{n}^{(m)} u_{n}+c_{n}^{(m)} v_{n}^{(m)}, \quad n \geq 1,
\end{array}\right\}
$$

where $\left\{v_{n}^{(1)}\right\}, \ldots,\left\{v_{n}^{(m)}\right\}$ are bounded sequences in $B$ and $\left\{a_{n}^{(i)}\right\},\left\{b_{n}^{(i)}\right\},\left\{c_{n}^{(i)}\right\}$ are appropriate real sequences in $[0,1]$ such that $a_{n}^{(i)}+b_{n}^{(i)}+c_{n}^{(i)}=1$ for each $i \in\{1,2, \ldots, m\}$.

The iterative procedure given by (1.3) is known as the multi-step Noor iterative procedure with errors (MNIPE). After Plubtieng and Wangkeeree [24], a numerous number of research articles have been published on different types of iterative procedures with errors for various kinds of mappings; see for instance [1, 9, 12, 25-27] and the references cited therein. Among the above-mentioned articles, Hussain et al. [1] studied the following special type of Ishikawa iterative procedure with errors (STIIPE) for two Lipschitz strictly hemicontractive-type mappings in arbitrary Banach spaces: For a given $u_{0} \in B$, the iterative sequences $\left\{u_{n}\right\}_{n=0}^{\infty}$ defined by

$$
\left.\begin{array}{l}
u_{n+1}=u_{n}^{(2)}=a_{n}^{(2)} u_{n}+b_{n}^{(2)} T u_{n}^{(1)}+c_{n}^{(2)} v_{n}^{(2)}, \\
u_{n}^{(1)}=a_{n}^{(1)} u_{n}+b_{n}^{(1)} S u_{n}+c_{n}^{(1)} v_{n}^{(1)}, \quad n \geq 0,
\end{array}\right\}
$$

where $\left\{v_{n}^{(1)}\right\},\left\{v_{n}^{(2)}\right\}$ are bounded sequences in $B$ and $\left\{a_{n}^{(i)}\right\},\left\{b_{n}^{(i)}\right\},\left\{c_{n}^{(i)}\right\}$ are appropriate real sequences in $[0,1]$ satisfying $a_{n}^{(i)}+b_{n}^{(i)}+c_{n}^{(i)}=1$ for all $i \in\{1,2\}$.

Stimulated by the work of Hussain et al. [1, 9], Plubtieng and Wangkeeree [24], Yu et al. [11], Agwu and Igbokwe [17] and Zegeye and Tufa [19] in this paper, we propose and study the following modified multi-step Noor iterative procedure with errors (MMNIPE) for two Lipschitz strictly hemicontractive-type mappings in arbitrary Banach spaces: For a given $u_{0} \in B$, and a fixed $m \in \mathbf{N}$, we compute the iterative sequences $\left\{u_{n}\right\}_{n=0}^{\infty}$ by

$$
\begin{aligned}
& u_{n+1}=u_{n}^{(m)}=a_{n}^{(m)} u_{n}+b_{n}^{(m)} T u_{n}^{(m-1)}+c_{n}^{(m)} v_{n}^{(m)}, \\
& u_{n}^{(m-1)}=a_{n}^{(m-1)} u_{n}+b_{n}^{(m-1)} T u_{n}^{(m-2)}+c_{n}^{(m-1)} v_{n}^{(m-1)}, \\
& \ldots \ldots \\
& u_{n}^{(2)}=a_{n}^{(2)} u_{n}+b_{n}^{(2)} T u_{n}^{(1)}+c_{n}^{(2)} v_{n}^{(2)}, \\
& u_{n}^{(1)}=a_{n}^{(1)} u_{n}+b_{n}^{(1)} S u_{n}+c_{n}^{(1)} v_{n}^{(1)}, \quad n \geq 0,
\end{aligned}
$$

where $\left\{v_{n}^{(1)}\right\}, \ldots,\left\{v_{n}^{(m)}\right\}$ are bounded sequences in $B$ and $\left\{a_{n}^{(i)}\right\},\left\{b_{n}^{(i)}\right\},\left\{c_{n}^{(i)}\right\}$ are appropriate real sequences in $[0,1]$ such that $a_{n}^{(i)}+b_{n}^{(i)}+c_{n}^{(i)}=1$ for each $i \in\{1,2, \ldots, m\}$. 
Remark 1.5 It is clear that the iterative procedures defined by (1.4) (the STIIPE given by Hussain et al. [1]), the Mann iterative procedure (MIP) given by Mann [28], the Ishikawa iterative procedure (IIP) given by Ishikawa [29], the Noor iterative procedure (NIP) given by $\mathrm{Xu}$ and Noor [30], Mann iterative procedures with errors (MIPE) given by Liu [31] and $\mathrm{Xu}$ [32], the Ishikawa iterative procedure with errors (IIPE) given by Liu [31] and Xu [32] and the three-step iterative procedure with errors (TIPE) given by Cho et al. [33] are all special cases of the newly proposed MMNIPE given by (1.5). That is, the iterative procedure defined by (1.5) is a general iterative procedure among the above-mentioned iterative procedures.

To the best of our knowledge, there does not exist any work about the convergence and almost common-stability and common-stability of the iterative procedure given by (1.5) for Lipschitz strictly hemicontractive-type mappings in arbitrary Banach spaces. From this context, here we establish the convergence, almost common-stability and common-stability of the newly proposed MMNIPE given by (1.5) for two Lipschitz strictly hemicontractive-type mappings in arbitrary Banach spaces. The rest of this paper is organized as follows:

In Sect. 2, we recall some essential definitions and fundamental results. Sect. 3 is the main part of this paper. Here, we establish convergence, almost common-stability and common-stability of our proposed MMNIPE given by (1.5). In Sect. 4, we discuss a numerical example to verify the main results of this paper. Finally, in Sect. 5, we conclude this paper.

\section{Preliminary notes}

This section is devoted to recalling some definitions and fundamental results which are truly needed to establish the main results.

Definition 2.1 (See $[4,34]$ ) The mapping $T$ is called pseudocontractive if the inequality

$$
\|q-r\| \leq\|q-r+t((I-T) q-(I-T) r)\|
$$

holds for each $q, r \in B$ and for all $t>0$. According to the result of Kato [35], it follows that $T$ is a pseudocontractive if and only if there exists a $h(q-r) \in J(q-r)$ such that

$$
\langle T q-\operatorname{Tr}, h(q-r)\rangle \leq\|q-r\|^{2}
$$

for all $q, r \in B . T$ is called strongly pseudocontractive if there exists a $t>1$ such that

$$
\|q-r\| \leq\left\|\left(1+t^{\prime}\right)(q-r)-t^{\prime} t(T q-T r)\right\|
$$

for all $q, r \in D_{T}$ and $t^{\prime}>0$. $T$ is called local strongly pseudocontractive if, for each $q \in D_{T}$, there exists a $t_{q}>1$ such that

$$
\|q-r\| \leq\left\|\left(1+t^{\prime}\right)(q-r)-t^{\prime} t_{q}(T q-T r)\right\|
$$

for all $q, r \in D_{T}$ and $t^{\prime}>0$. 
Definition 2.2 (See [36-38]) Suppose $u_{0} \in B$ and $u_{n+1}=f\left(u_{n}, T\right)$ defines an iterative procedure which yields a sequence of points $\left\{u_{n}\right\} \subset B$. Let $F_{T} \neq \varphi$ and let $\left\{u_{n}\right\}$ converge to a fixed point $q$ of $T$. Let $\left\{v_{n}\right\} \subset B$ and $\left\{\delta_{n}\right\}$ be a sequence in $[0, \infty)$, where $\delta_{n}=\left\|v_{n+1}-f\left(v_{n}, T\right)\right\|$. Now, if $\lim _{n \rightarrow \infty} \delta_{n}=0$ implies that $\lim _{n \rightarrow \infty} v_{n}=q$, then the iterative procedure defined by $u_{n+1}=f\left(u_{n}, T\right)$ is said to be $T$-stable or stable on $B$ with respect to $T$ and if $\sum_{n=0}^{\infty} \delta_{n}<\infty$ implies that $\lim _{n \rightarrow \infty} v_{n}=q$, then the iterative procedure defined by $u_{n+1}=f\left(u_{n}, T\right)$ is said to be an almost $T$-stable on $B$ with respect to $T$.

Definition 2.3 (See [1]) Let $B$ be a nonempty convex subset of an arbitrary Banach space $X$ and let $T$ and $S$ be two self-operators on $B$. Suppose $u_{0} \in B$ and $u_{n+1}=f\left(u_{n}, T, S\right)$ defines an iterative procedure which yields a sequence of points $\left\{u_{n}\right\} \subset B$. Let $F_{T} \cap F_{S} \neq \varphi$ and let $\left\{u_{n}\right\}$ converges strongly to a common fixed point $q$ of $T$ and $S$. Let $\left\{v_{n}\right\}$ be any bounded sequence in $B$ and $\left\{\mu_{n}\right\}$ be a sequence in $[0, \infty)$, where $\mu_{n}=\left\|v_{n+1}-f\left(v_{n}, T, S\right)\right\|$. Now, if $\lim _{n \rightarrow \infty} \mu_{n}=0$ implies that $\lim _{n \rightarrow \infty} v_{n}=r$, then the iterative procedure defined by $u_{n+1}=$ $f\left(u_{n}, T, S\right)$ is said to be a common-stable on $B$ and if $\sum_{n=0}^{\infty} \mu_{n}<\infty$ implies that $\lim _{n \rightarrow \infty} v_{n}=$ $q$, then the iterative procedure defined by $u_{n+1}=f\left(u_{n}, T, S\right)$ is said to be an almost commonstable on $B$.

Now, we recall some lemmas which are essential to prove the main results of this paper.

Lemma 2.4 (See [39]) Let $\left\{\alpha_{n}\right\}_{n=0}^{\infty},\left\{\beta_{n}\right\}_{n=0}^{\infty},\left\{\gamma_{n}\right\}_{n=0}^{\infty}$ and $\left\{\omega_{n}\right\}_{n=0}^{\infty}$ be nonnegative real sequences such that

$$
\alpha_{n+1} \leq\left(1-\omega_{n}\right) \alpha_{n}+\omega_{n} \beta_{n}+\gamma_{n}, \quad n \geq 0,
$$

with $\left\{\omega_{n}\right\}_{n=0}^{\infty} \subset[0,1], \sum_{n=0}^{\infty} \omega_{n}=\infty, \sum_{n=0}^{\infty} \gamma_{n}<\infty$ and $\lim _{n \rightarrow \infty} \beta_{n}=0$. Then $\lim _{n \rightarrow \infty} \alpha_{n}=0$.

Lemma 2.5 (See [40]) Let $\left\{\alpha_{n}\right\}_{n=0}^{\infty}$ and $\left\{\beta_{n}\right\}_{n=0}^{\infty}$ be sequences of nonnegative real numbers and $0 \leq \eta<1$, so that

$$
\alpha_{n+1} \leq \eta \alpha_{n}+\beta_{n}, \quad \forall n \geq 0 .
$$

(i) If $\lim _{n \rightarrow \infty} \beta_{n}=0$, then $\lim _{n \rightarrow \infty} \alpha_{n}=0$.

(ii) If $\sum_{n=0}^{\infty} \beta_{n}<\infty$, then $\sum_{n=0}^{\infty} \alpha_{n}<\infty$.

Lemma 2.6 (See [35]) Let $x, y \in X$. Then $\|x\| \leq\|x+r y\|$ for every $r>0$ if and only if there is $f \in J(x)$ such that $\operatorname{Re}(y, f) \geq 0$.

Lemma 2.7 (See [4]) Let $T: D_{T} \subseteq X \rightarrow X$ be an operator with $F_{T} \neq \varphi$. Then $T$ is strictly hemicontractive if and if only if there exists a $t>1$ such that for all $x \in D_{T}$ and $q \in F_{T}$ there exists $h \in J(x-q)$ satisfying

$$
\operatorname{Re}\langle x-T x, h(x-q)\rangle \geq\left(1-\frac{1}{t}\right)\|x-q\|^{2}
$$

Lemma 2.8 (See [12]) Let $X$ be an arbitrary norm linear space and $T: D_{T} \subseteq X \rightarrow X$ be an operator. 
(a) If $T$ is a local strongly pseudocontractive operator and $F_{T} \neq \varphi$, then $F_{T}$ is a singleton and $T$ is strictly hemicontractive.

(b) If $T$ is strictly hemicontractive, then $F_{T}$ is a singleton.

\section{Convergence and stability of modified multi-step Noor iterative procedure with errors}

In this section, we state and prove the convergence and stability of our proposed MMNIPE for two Lipschitz strictly hemicontractive-type mappings.

Let $\lambda=\frac{\sigma-1}{\sigma} \in(0,1)$, where $\sigma>1, L$ be a common Lipschitz constant of two strictly hemicontractive-type mappings $T, S$ and $I$ be an identity mapping on the arbitrary Banach space $X$. In the above-mentioned context, we state and prove the following theorems.

Theorem 3.1 Let B be a nonempty closed convex subset of $X$ and $T$ and $S$ be two Lipschitz strictly hemicontractive-type mappings from $B$ into itself. Suppose that $\left\{v_{n}^{(1)}\right\}, \ldots,\left\{v_{n}^{(m)}\right\}$ are arbitrary bounded sequences in $B$ and $\left\{a_{n}^{(i)}\right\},\left\{b_{n}^{(i)}\right\},\left\{c_{n}^{(i)}\right\}$ for each $i \in\{1,2, \ldots, m\}$ are any appropriate real sequences in $[0,1]$ satisfying the following conditions:

(1) $a_{n}^{(i)}+b_{n}^{(i)}+c_{n}^{(i)}=1$, for each $i \in\{1,2,3, \ldots, m\}$,

(2) $c_{n}^{(m)}=\mathrm{o}\left(b_{n}^{(m)}\right)$

(3) $\lim _{n \rightarrow \infty} c_{n}^{(j)}=0$, for each $j \in\{1,2,3, \ldots, m-1\}$,

(4) $\sum_{n=0}^{\infty} b_{n}^{(j)}=\infty$ for each $j \in\{2,3, \ldots, m\}$,

(5)

$$
\begin{aligned}
L[ & (1+L) b_{n}^{(m)}+(1+L)^{2} b_{n}^{(m-1)}+L(1+L)^{2} b_{n}^{(m-1)} b_{n}^{(m-2)} \\
& +L^{2}(1+L)^{2} b_{n}^{(m-1)} b_{n}^{(m-2)} b_{n}^{(m-3)}+\cdots+L^{m-3}(1+L)^{2} b_{n}^{(m-1)} b_{n}^{(m-2)} b_{n}^{(m-3)} \cdots b_{n}^{(2)} \\
& +L^{m-2}(1+L)^{2} b_{n}^{(m-1)} b_{n}^{(m-2)} b_{n}^{(m-3)} \cdots b_{n}^{(2)} b_{n}^{(1)} \\
& +\left[c_{n}^{(m)}+(1+L) c_{n}^{(m-1)}+L(1+L) b_{n}^{(m-1)} c_{n}^{(m-2)}\right. \\
& +L^{2}(1+L) b_{n}^{(m-1)} b_{n}^{(m-2)} c_{n}^{(m-3)}+\cdots+L^{m-3}(1+L) b_{n}^{(m-1)} b_{n}^{(m-2)} \cdots b_{n}^{(3)} c_{n}^{(2)} \\
& \left.\left.+L^{m-2}(1+L) b_{n}^{(m-1)} b_{n}^{(m-2)} b_{n}^{(m-3)} \cdots b_{n}^{(2)} c_{n}^{(1)}\right]\right]+\frac{c_{n}^{(m)}}{b_{n}^{(m)}} \leq \lambda(\lambda-\theta), \quad n \geq 0,
\end{aligned}
$$

where $\theta$ is a constant in $(0, \lambda)$ and $\lambda \in(0,1)$.

Assume an iterative sequence $\left\{u_{n}\right\}_{n=0}^{\infty}$ defined by (1.5). Let $\left\{w_{n}\right\}_{n=0}^{\infty}$ be any sequence in $B$ and $\left\{\mu_{n}\right\}_{n=0}^{\infty}$ be a sequence defined by

$$
\mu_{n}=\left\|w_{n+1}-x_{n}\right\|, \quad n \geq 0,
$$

where

$$
\begin{aligned}
& x_{n}=x_{n}^{(m)}=a_{n}^{(m)} w_{n}+b_{n}^{(m)} T x_{n}^{(m-1)}+c_{n}^{(m)} v_{n}^{(m)}, \\
& x_{n}^{(m-1)}=a_{n}^{(m-1)} w_{n}+b_{n}^{(m-1)} T x_{n}^{(m-2)}+c_{n}^{(m-1)} v_{n}^{(m-1)}, \\
& \cdots \cdots \\
& x_{n}^{(2)}=a_{n}^{(2)} w_{n}+b_{n}^{(2)} T x_{n}^{(1)}+c_{n}^{(2)} v_{n}^{(2)}, \\
& x_{n}^{(1)}=a_{n}^{(1)} w_{n}+b_{n}^{(1)} S w_{n}+c_{n}^{(1)} v_{n}^{(1)}, \quad n \geq 0 .
\end{aligned}
$$


(i) the iterative sequence $\left\{u_{n}\right\}_{n=0}^{\infty}$ given by (1.5) converges strongly to the common fixed $r$ of $T$ and $S$ and the following inequality holds:

$$
\begin{aligned}
\| u_{n+1} & -r \| \\
\leq & \left(1-\theta b_{n}^{(m)}\right)\left\|u_{n}-r\right\|+\lambda^{-1}(1+L)\left[c_{n}^{(m)}\left\|v_{n}^{(m)}-r\right\|+c_{n}^{(m-1)} L b_{n}^{(m)}\left\|v_{n}^{(m-1)}-r\right\|\right. \\
& +c_{n}^{(m-2)} L^{2} b_{n}^{(m)} b_{n}^{(m-1)}\left\|v_{n}^{(m-2)}-r\right\|+c_{n}^{(m-3)} L^{3} b_{n}^{(m)} b_{n}^{(m-1)} b_{n}^{(m-2)}\left\|v_{n}^{(m-3)}-r\right\| \\
& +\cdots+c_{n}^{(2)} L^{m-2} b_{n}^{(m)} b_{n}^{(m-1)} b_{n}^{(m-2)} \cdots b_{n}^{(3)}\left\|v_{n}^{(2)}-r\right\| \\
& \left.+c_{n}^{(1)} L^{m-1} b_{n}^{(m)} b_{n}^{(m-1)} b_{n}^{(m-2)} \cdots b_{n}^{(2)}\left\|v_{n}^{(1)}-r\right\|\right], \quad n \geq 0,
\end{aligned}
$$

(ii)

$$
\begin{aligned}
\| w_{n+1} & -r \| \\
\leq & \left(1-\theta b_{n}^{(m)}\right)\left\|w_{n}-r\right\| \\
& +\lambda^{-1}(1+L)\left[c_{n}^{(m)}\left\|v_{n}^{(m)}-r\right\|+L\left[b_{n}^{(m)} c_{n}^{(m-1)}\left\|v_{n}^{(m-1)}-r\right\|\right.\right. \\
& +L\left[b_{n}^{(m)} b_{n}^{(m-1)} c_{n}^{(m-2)}\left\|v_{n}^{(m-2)}-r\right\|+L\left[b_{n}^{(m)} b_{n}^{(m-1)} b_{n}^{(m-2)} c_{n}^{(m-3)}\left\|v_{n}^{(m-3)}-r\right\|\right.\right. \\
& +\cdots+L\left[b_{n}^{(m)} b_{n}^{(m-1)} b_{n}^{(m-2)} \cdots b_{n}^{(3)} c_{n}^{(2)}\left\|v_{n}^{(2)}-r\right\|\right. \\
& \left.\left.\left.\left.\left.+L\left[b_{n}^{(m)} b_{n}^{(m-1)} b_{n}^{(m-2)} b_{n}^{(m-3)} \cdots b_{n}^{(2)} c_{n}^{(1)}\left\|v_{n}^{(1)}-r\right\|\right]\right]\right]\right] \cdots\right]\right]+\mu_{n}, \quad n \geq 0,
\end{aligned}
$$

(iii) $\sum_{n=0}^{\infty} \mu_{n}<\infty$ implies that $\lim _{n \rightarrow \infty} w_{n}=r$, so that $\left\{u_{n}^{(m)}\right\}_{n=0}^{\infty}$ is almost common-stable on $B$,

(iv) $\lim _{n \rightarrow \infty} w_{n}=r$, implies that $\lim _{n \rightarrow \infty} \mu_{n}=0$.

Proof (i) From the condition (2), we obtain $c_{n}^{(m)}=\delta_{n} b_{n}^{(m)}$, and $\delta_{n} \rightarrow 0$ as $n \rightarrow \infty$. By an application of Lemma 2.8, we see that $F_{T} \cap F_{S}$ is singleton, and let $F_{T} \cap F_{S}=\{r\}$ for some $r \in B$. Put

$$
A=\max \left\{\sup _{n \geq 0}\left\{\left\|v_{n}^{(1)}-r\right\|\right\}, \sup _{n \geq 0}\left\{\left\|v_{n}^{(2)}-r\right\|\right\}, \sup _{n \geq 0}\left\{\left\|v_{n}^{(3)}-r\right\|\right\}, \ldots, \sup _{n \geq 0}\left\{\left\|v_{n}^{(m)}-r\right\|\right\}\right\} .
$$

Since $T$ is strictly hemicontractive, from Lemma 2.7, we obtain

$$
\begin{aligned}
& \operatorname{Re}\langle x-T x, h(x-r)\rangle \geq \lambda\|x-r\|^{2} \\
& \Rightarrow \quad \operatorname{Re}\langle(I-T-\lambda I) x-(I-T-\lambda I) r, h(x-r)\rangle \geq 0, \quad \forall x \in B .
\end{aligned}
$$

Now, from (3.2) and Lemma 2.6, we have

$$
\|x-r\| \leq\|x-r+q[(I-T-\lambda I) x-(I-T-\lambda I) r]\|, \quad \forall x \in B \text {, and } \forall q>0 .
$$

Also, from the first equation of (1.5), we get

$$
\begin{aligned}
u_{n+1}=a_{n}^{(m)} u_{n}+b_{n}^{(m)} & T u_{n}^{(m-1)}+c_{n}^{(m)} v_{n}^{(m)} \\
\Rightarrow \quad\left(1-b_{n}^{(m)}\right) u_{n}= & \left(1-(1-\lambda) b_{n}^{(m)}\right) u_{n+1}+b_{n}^{(m)}(I-T-\lambda I) u_{n+1} \\
& +b_{n}^{(m)}\left(T u_{n+1}-T u_{n}^{(m-1)}\right)-c_{n}^{(m)}\left(v_{n}^{(m)}-u_{n}\right)
\end{aligned}
$$


and since $r \in B$ is the fixed point of $T$, it follows that

$$
\left(1-b_{n}^{(m)}\right) r=\left(1-(1-\lambda) b_{n}^{(m)}\right) r+b_{n}^{(m)}(I-T-\lambda I) r .
$$

Now, for all $n \geq 0$ from (3.4) and (3.5), we have

$$
\begin{aligned}
(1- & \left.b_{n}^{(m)}\right)\left\|u_{n}-r\right\| \\
\geq & \left\|\left(1-(1-\lambda) b_{n}^{(m)}\right)\left(u_{n+1}-r\right)+b_{n}^{(m)}(I-T-\lambda I)\left(u_{n+1}-r\right)\right\| \\
& \quad-b_{n}^{(m)}\left\|T u_{n+1}-T u_{n}^{(m-1)}\right\|-c_{n}^{(m)}\left\|v_{n}^{(m)}-u_{n}\right\| \\
= & \left(1-(1-\lambda) b_{n}^{(m)}\right)\left\|u_{n+1}-r+\frac{b_{n}^{(m)}}{1-(1-\lambda) b_{n}^{(m)}}(I-T-\lambda I)\left(u_{n+1}-r\right)\right\| \\
& \quad-b_{n}^{(m)}\left\|T u_{n+1}-T u_{n}^{(m-1)}\right\|-c_{n}^{(m)}\left\|v_{n}^{(m)}-u_{n}\right\| \\
\geq & \left(1-(1-\lambda) b_{n}^{(m)}\right)\left\|u_{n+1}-r\right\|-b_{n}^{(m)}\left\|T u_{n+1}-T u_{n}^{(m-1)}\right\|-c_{n}^{(m)}\left\|v_{n}^{(m)}-u_{n}\right\|
\end{aligned}
$$

which implies that

$$
\begin{aligned}
\left\|u_{n+1}-r\right\| & \\
\leq & \frac{1-b_{n}^{(m)}}{1-(1-\lambda) b_{n}^{(m)}}\left\|u_{n}-r\right\|+\frac{b_{n}^{(m)}}{1-(1-\lambda) b_{n}^{(m)}}\left\|T u_{n+1}-T u_{n}^{(m-1)}\right\| \\
& +\frac{c_{n}^{(m)}}{1-(1-\lambda) b_{n}^{(m)}}\left\|v_{n}^{(m)}-u_{n}\right\| \\
\leq & \left(1-\lambda b_{n}^{(m)}\right)\left\|u_{n}-r\right\|+\lambda^{-1} b_{n}^{(m)}\left\|T u_{n+1}-T u_{n}^{(m-1)}\right\|+\lambda^{-1} c_{n}^{(m)}\left\|v_{n}^{(m)}-u_{n}\right\| \\
\leq & \left(1-\lambda b_{n}^{(m)}\right)\left\|u_{n}-r\right\|+\lambda^{-1} L b_{n}^{(m)}\left\|u_{n+1}-u_{n}^{(m-1)}\right\|+\lambda^{-1} c_{n}^{(m)}\left\|v_{n}^{(m)}-u_{n}\right\| \\
\leq & \left(1-\lambda b_{n}^{(m)}\right)\left\|u_{n}-r\right\|+\lambda^{-1} L b_{n}^{(m)}\left\|u_{n+1}-u_{n}^{(m-1)}\right\|+\lambda^{-1} c_{n}^{(m)}\left(\left\|v_{n}^{(m)}-r\right\|+\left\|u_{n}-r\right\|\right) \\
= & \left(1-\lambda b_{n}^{(m)}+\lambda^{-1} c_{n}^{(m)}\right)\left\|u_{n}-r\right\|+\lambda^{-1} L b_{n}^{(m)}\left\|u_{n+1}-u_{n}^{(m-1)}\right\| \\
& +\lambda^{-1} c_{n}^{(m)}\left\|v_{n}^{(m)}-r\right\| .
\end{aligned}
$$

Again, from (1.5), we get

$$
\begin{aligned}
&\left\|u_{n+1}-u_{n}^{(m-1)}\right\| \\
&=\left\|a_{n}^{(m)} u_{n}+b_{n}^{(m)} T u_{n}^{(m-1)}+c_{n}^{(m)} v_{n}^{(m)}-a_{n}^{(m-1)} u_{n}-b_{n}^{(m-1)} T u_{n}^{(m-2)}-c_{n}^{(m-1)} v_{n}^{(m-1)}\right\| \\
&=\|\left(1-b_{n}^{(m)}-c_{n}^{(m)}\right) u_{n}+b_{n}^{(m)} T u_{n}^{(m-1)}+c_{n}^{(m)} v_{n}^{(m)}-\left(1-b_{n}^{(m-1)}-c_{n}^{(m-1)}\right) u_{n} \\
& \quad-b_{n}^{(m-1)} T u_{n}^{(m-2)}-c_{n}^{(m-1)} v_{n}^{(m-1)} \| \\
&=\left\|b_{n}^{(m)}\left(T u_{n}^{(m-1)}-u_{n}\right)+c_{n}^{(m)}\left(v_{n}^{(m)}-u_{n}\right)+b_{n}^{(m-1)}\left(u_{n}-T u_{n}^{(m-2)}\right)-c_{n}^{(m-1)}\left(v_{n}^{(m-1)}-u_{n}\right)\right\| \\
& \leq\left\|b_{n}^{(m)}\left(T u_{n}^{(m-1)}-u_{n}\right)+c_{n}^{(m)}\left(v_{n}^{(m)}-u_{n}\right)\right\| \\
& \quad+\left\|b_{n}^{(m-1)}\left(u_{n}-T u_{n}^{(m-2)}\right)-c_{n}^{(m-1)}\left(v_{n}^{(m-1)}-u_{n}\right)\right\| \\
& \leq b_{n}^{(m)}\left\|u_{n}-T u_{n}^{(m-1)}\right\|+c_{n}^{(m)}\left\|v_{n}^{(m)}-u_{n}\right\|+b_{n}^{(m-1)}\left\|u_{n}-T u_{n}^{(m-2)}\right\| \\
&+c_{n}^{(m-1)}\left\|v_{n}^{(m-1)}-u_{n}\right\|
\end{aligned}
$$




$$
\begin{aligned}
\leq & b_{n}^{(m)}\left(\left\|u_{n}-r\right\|+\left\|r-T u_{n}^{(m-1)}\right\|\right)+c_{n}^{(m)}\left(\left\|v_{n}^{(m)}-r\right\|+\left\|u_{n}-r\right\|\right) \\
& +b_{n}^{(m-1)}\left(\left\|u_{n}-r\right\|+\left\|r-T u_{n}^{(m-2)}\right\|\right)+c_{n}^{(m-1)}\left(\left\|v_{n}^{(m-1)}-r\right\|+\left\|u_{n}-r\right\|\right) \\
\leq & b_{n}^{(m)}\left(\left\|u_{n}-r\right\|+L\left\|u_{n}^{(m-1)}-r\right\|\right)+c_{n}^{(m)}\left(\left\|v_{n}^{(m)}-r\right\|+\left\|u_{n}-r\right\|\right) \\
& +b_{n}^{(m-1)}\left(\left\|u_{n}-r\right\|+L\left\|u_{n}^{(m-2)}-r\right\|\right)+c_{n}^{(m-1)}\left(\left\|v_{n}^{(m-1)}-r\right\|+\left\|u_{n}-r\right\|\right) \\
= & {\left[b_{n}^{(m)}+b_{n}^{(m-1)}+c_{n}^{(m)}+c_{n}^{(m-1)}\right]\left\|u_{n}-r\right\|+b_{n}^{(m)} L\left\|u_{n}^{(m-1)}-r\right\|+b_{n}^{(m-1)} L\left\|u_{n}^{(m-2)}-r\right\| } \\
& +c_{n}^{(m)}\left\|v_{n}^{(m)}-r\right\|+c_{n}^{(m-1)}\left\|v_{n}^{(m-1)}-r\right\| .
\end{aligned}
$$

But

$$
\begin{aligned}
\left\|u_{n}^{(m-1)}-r\right\|= & \left\|a_{n}^{(m-1)} u_{n}+b_{n}^{(m-1)} T u_{n}^{(m-2)}+c_{n}^{(m-1)} v_{n}^{(m-1)}-r\right\| \\
= & \left\|\left(1-b_{n}^{(m-1)}-c_{n}^{(m-1)}\right) u_{n}+b_{n}^{(m-1)} T u_{n}^{(m-2)}+c_{n}^{(m-1)} v_{n}^{(m-1)}-r\right\| \\
= & \left\|u_{n}-r+b_{n}^{(m-1)}\left(T u_{n}^{(m-2)}-r+r-u_{n}\right)+c_{n}^{(m-1)}\left(v_{n}^{(m-1)}-r+r-u_{n}\right)\right\| \\
\leq & \left\|u_{n}-r\right\|+b_{n}^{(m-1)}\left\|T u_{n}^{(m-2)}-r\right\|+b_{n}^{(m-1)}\left\|u_{n}-r\right\|+c_{n}^{(m-1)}\left\|v_{n}^{(m-1)}-r\right\| \\
& \quad+c_{n}^{(m-1)}\left\|u_{n}-r\right\| \\
\leq & \left\|u_{n}-r\right\|+b_{n}^{(m-1)} L\left\|u_{n}^{(m-2)}-r\right\|+b_{n}^{(m-1)}\left\|u_{n}-r\right\|+c_{n}^{(m-1)}\left\|v_{n}^{(m-1)}-r\right\| \\
& \quad+c_{n}^{(m-1)}\left\|u_{n}-r\right\| \\
= & {\left[1+b_{n}^{(m-1)}+c_{n}^{(m-1)}\right]\left\|u_{n}-r\right\|+b_{n}^{(m-1)} L\left\|u_{n}^{(m-2)}-r\right\| } \\
& +c_{n}^{(m-1)}\left\|v_{n}^{(m-1)}-r\right\| .
\end{aligned}
$$

Substituting (3.8) in (3.7), we have

$$
\begin{aligned}
\| u_{n+1} & -u_{n}^{(m-1)} \| \\
\leq & {\left[b_{n}^{(m)}+b_{n}^{(m-1)}+c_{n}^{(m)}+c_{n}^{(m-1)}\right]\left\|u_{n}-r\right\| } \\
& +b_{n}^{(m)} L\left[\left[1+b_{n}^{(m-1)}+c_{n}^{(m-1)}\right]\left\|u_{n}-r\right\|+b_{n}^{(m-1)} L\left\|u_{n}^{(m-2)}-r\right\|+c_{n}^{(m-1)}\left\|v_{n}^{(m-1)}-r\right\|\right] \\
& +b_{n}^{(m-1)} L\left\|u_{n}^{(m-2)}-r\right\|+c_{n}^{(m)}\left\|v_{n}^{(m)}-r\right\|+c_{n}^{(m-1)}\left\|v_{n}^{(m-1)}-r\right\| \\
= & \left.b_{n}^{(m)}+b_{n}^{(m-1)}+b_{n}^{(m)} L\left[1+b_{n}^{(m-1)}+c_{n}^{(m-1)}\right]+c_{n}^{(m)}+c_{n}^{(m-1)}\right]\left\|u_{n}-r\right\| \\
& +\left[b_{n}^{(m)} b_{n}^{(m-1)} L^{2}+b_{n}^{(m-1)} L\right]\left\|u_{n}^{(m-2)}-r\right\|+c_{n}^{(m)}\left\|v_{n}^{(m)}-r\right\| \\
& +\left[b_{n}^{(m)} L c_{n}^{(m-1)}+c_{n}^{(m-1)}\right]\left\|v_{n}^{(m-1)}-r\right\| \\
= & \left.(1+L) b_{n}^{(m)}+\left(1+L b_{n}^{(m)}\right) b_{n}^{(m-1)}+c_{n}^{(m)}+\left(1+L b_{n}^{(m)}\right) c_{n}^{(m-1)}\right]\left\|u_{n}-r\right\| \\
& +L b_{n}^{(m-1)}\left(1+L b_{n}^{(m)}\right)\left\|u_{n}^{(m-2)}-r\right\|+c_{n}^{(m)}\left\|v_{n}^{(m)}-r\right\| \\
& +c_{n}^{(m-1)}\left(1+L b_{n}^{(m)}\right)\left\|v_{n}^{(m-1)}-r\right\| .
\end{aligned}
$$

But, if we replace $m$ by $m-1$ in (3.8), then we have

$$
\begin{aligned}
\left\|u_{n}^{(m-2)}-r\right\|= & {\left[1+b_{n}^{(m-2)}+c_{n}^{(m-2)}\right]\left\|u_{n}-r\right\|+b_{n}^{(m-2)} L\left\|u_{n}^{(m-3)}-r\right\| } \\
& +c_{n}^{(m-2)}\left\|v_{n}^{(m-2)}-r\right\| .
\end{aligned}
$$


Now, substituting (3.10) in (3.9), we have

$$
\begin{aligned}
\| u_{n+1} & -u_{n}^{(m-1)} \| \\
\leq & {\left[(1+L) b_{n}^{(m)}+\left(1+L b_{n}^{(m)}\right) b_{n}^{(m-1)}+c_{n}^{(m)}+\left(1+L b_{n}^{(m)}\right) c_{n}^{(m-1)}\right]\left\|u_{n}-r\right\| } \\
& +L\left(1+L b_{n}^{(m)}\right) b_{n}^{(m-1)}\left[\left[1+b_{n}^{(m-2)}+c_{n}^{(m-2)}\right]\left\|u_{n}-r\right\|+b_{n}^{(m-2)} L\left\|u_{n}^{(m-3)}-r\right\|\right. \\
& \left.+c_{n}^{(m-2)}\left\|v_{n}^{(m-2)}-r\right\|\right]+c_{n}^{(m)}\left\|v_{n}^{(m)}-r\right\|+c_{n}^{(m-1)}\left(1+L b_{n}^{(m)}\right)\left\|v_{n}^{(m-1)}-r\right\| \\
\leq & {\left[(1+L) b_{n}^{(m)}+(1+L)\left(1+L b_{n}^{(m)}\right) b_{n}^{(m-1)}+L\left(1+L b_{n}^{(m)}\right) b_{n}^{(m-1)} b_{n}^{(m-2)}+c_{n}^{(m)}\right.} \\
& \left.+\left(1+L b_{n}^{(m)}\right) c_{n}^{(m-1)}+L\left(1+L b_{n}^{(m)}\right) b_{n}^{(m-1)} c_{n}^{(m-2)}\right]\left\|u_{n}-r\right\| \\
& +L^{2}\left(1+L b_{n}^{(m)}\right) b_{n}^{(m-1)} b_{n}^{(m-2)}\left\|u_{n}^{(m-3)}-r\right\|+c_{n}^{(m)}\left\|v_{n}^{(m)}-r\right\| \\
& +c_{n}^{(m-1)}\left(1+L b_{n}^{(m)}\right)\left\|v_{n}^{(m-1)}-r\right\|+c_{n}^{(m-2)} L b_{n}^{(m-1)}\left(1+L b_{n}^{(m)}\right)\left\|v_{n}^{(m-2)}-r\right\| .
\end{aligned}
$$

But, if we replace $m$ by $m-1$ in (3.10), then we have

$$
\begin{aligned}
\left\|u_{n}^{(m-3)}-r\right\|= & {\left[1+b_{n}^{(m-3)}+c_{n}^{(m-3)}\right]\left\|u_{n}-r\right\|+b_{n}^{(m-3)} L\left\|u_{n}^{(m-4)}-r\right\| } \\
& +c_{n}^{(m-3)}\left\|v_{n}^{(m-3)}-r\right\| .
\end{aligned}
$$

Substituting (3.12) in (3.11), we have

$$
\begin{aligned}
\| u_{n+1} & -u_{n}^{(m-1)} \| \\
\leq & {\left[(1+L) b_{n}^{(m)}+(1+L)\left(1+L b_{n}^{(m)}\right) b_{n}^{(m-1)}+L\left(1+L b_{n}^{(m)}\right) b_{n}^{(m-1)} b_{n}^{(m-2)}+c_{n}^{(m)}\right.} \\
& \left.+\left(1+L b_{n}^{(m)}\right) c_{n}^{(m-1)}+L\left(1+L b_{n}^{(m)}\right) b_{n}^{(m-1)} c_{n}^{(m-2)}\right]\left\|u_{n}-r\right\| \\
& +L^{2}\left(1+L b_{n}^{(m)}\right) b_{n}^{(m-1)} b_{n}^{(m-2)}\left[\left[1+b_{n}^{(m-3)}+c_{n}^{(m-3)}\right]\left\|u_{n}-r\right\|\right. \\
& \left.+L b_{n}^{(m-3)}\left\|u_{n}^{(m-4)}-r\right\|+c_{n}^{(m-3)}\left\|v_{n}^{(m-3)}-r\right\|\right]+c_{n}^{(m)}\left\|v_{n}^{(m)}-r\right\| \\
& +c_{n}^{(m-1)}\left(1+L b_{n}^{(m)}\right)\left\|v_{n}^{(m-1)}-r\right\|+c_{n}^{(m-2)} L\left(1+L b_{n}^{(m)}\right) b_{n}^{(m-1)}\left\|v_{n}^{(m-2)}-r\right\| \\
= & (1+L) b_{n}^{(m)}+(1+L)\left(1+L b_{n}^{(m)}\right) b_{n}^{(m-1)}+L(1+L)\left(1+L b_{n}^{(m)}\right) b_{n}^{(m-1)} b_{n}^{(m-2)} \\
& +L^{2}\left(1+L b_{n}^{(m)}\right) b_{n}^{(m-1)} b_{n}^{(m-2)} b_{n}^{(m-3)}+c_{n}^{(m)}+\left(1+L b_{n}^{(m)}\right) c_{n}^{(m-1)} \\
& \left.+L\left(1+L b_{n}^{(m)}\right) b_{n}^{(m-1)} c_{n}^{(m-2)}+L^{2}\left(1+L b_{n}^{(m)}\right) b_{n}^{(m-1)} b_{n}^{(m-2)} c_{n}^{(m-3)}\right]\left\|u_{n}-q\right\| \\
& +L^{3}\left(1+L b_{n}^{(m)}\right) b_{n}^{(m-1)} b_{n}^{(m-2)} b_{n}^{(m-3)}\left\|u_{n}^{(m-4)}-r\right\| \\
& +c_{n}^{(m)}\left\|v_{n}^{(m)}-r\right\|+c_{n}^{(m-1)}\left(1+L b_{n}^{(m)}\right)\left\|v_{n}^{(m-1)}-r\right\| \\
& +c_{n}^{(m-2)} L\left(1+L b_{n}^{(m)}\right) b_{n}^{(m-1)}\left\|v_{n}^{(m-2)}-r\right\| \\
& +c_{n}^{(m-3)} L^{2}\left(1+L b_{n}^{(m)}\right) b_{n}^{(m-1)} b_{n}^{(m-2)}\left\|v_{n}^{(m-3)}-r\right\| .
\end{aligned}
$$

Continuing the above procedure up to second iterative step of (1.5), we obtain

$$
\begin{aligned}
& \left\|u_{n+1}-u_{n}^{(m-1)}\right\| \\
& \leq\left[(1+L) b_{n}^{(m)}+(1+L)\left(1+L b_{n}^{(m)}\right) b_{n}^{(m-1)}+L(1+L)\left(1+L b_{n}^{(m)}\right) b_{n}^{(m-1)} b_{n}^{(m-2)}\right. \\
& \quad+L^{2}\left(1+L b_{n}^{(m)}\right) b_{n}^{(m-1)} b_{n}^{(m-2)} b_{n}^{(m-3)}+\cdots+L^{m-3}\left(1+L b_{n}^{(m)}\right) b_{n}^{(m-1)} b_{n}^{(m-2)} b_{n}^{(m-3)} \cdots b_{n}^{(2)}
\end{aligned}
$$




$$
\begin{aligned}
& +\left[c_{n}^{(m)}+\left(1+L b_{n}^{(m)}\right) c_{n}^{(m-1)}+L\left(1+L b_{n}^{(m)}\right) b_{n}^{(m-1)} c_{n}^{(m-2)}\right. \\
& +L^{2}\left(1+L b_{n}^{(m)}\right) b_{n}^{(m-1)} b_{n}^{(m-2)} c_{n}^{(m-3)} \\
& \left.\left.+\cdots+L^{m-3}\left(1+L b_{n}^{(m)}\right) b_{n}^{(m-1)} b_{n}^{(m-2)} \cdots b_{n}^{(2)} c_{n}^{(3)}\right]\right]\left\|u_{n}-r\right\| \\
& +L^{m-2}\left(1+L b_{n}^{(m)}\right) b_{n}^{(m-1)} b_{n}^{(m-2)} b_{n}^{(m-3)} \cdots b_{n}^{(2)}\left\|u_{n}^{(1)}-r\right\|+\left[c_{n}^{(m)}\left\|v_{n}^{(m)}-r\right\|\right. \\
& +c_{n}^{(m-1)}\left(1+L b_{n}^{(m)}\right)\left\|v_{n}^{(m-1)}-r\right\|+c_{n}^{(m-2)} L\left(1+L b_{n}^{(m)}\right) b_{n}^{(m-1)}\left\|v_{n}^{(m-2)}-r\right\| \\
& +c_{n}^{(m-3)} L^{2}\left(1+L b_{n}^{(m)}\right) b_{n}^{(m-1)} b_{n}^{(m-2)}\left\|v_{n}^{(m-3)}-r\right\| \\
& \left.+\cdots+c_{n}^{(2)} L^{m-3}\left(1+L b_{n}^{(m)}\right) b_{n}^{(m-1)} b_{n}^{(m-2)} \cdots b_{n}^{(3)}\left\|v_{n}^{(2)}-r\right\|\right] .
\end{aligned}
$$

Now, from the last equation of (1.5), we have

$$
\begin{aligned}
\left\|u_{n}^{(1)}-r\right\| & =\left\|a_{n}^{(1)} u_{n}+b_{n}^{(1)} S u_{n}+c_{n}^{(1)} v_{n}^{(1)}-r\right\| \\
& \leq\left\|u_{n}-r\right\|+b_{n}^{(1)}\left\|S u_{n}-r\right\|+b_{n}^{(1)}\left\|u_{n}-r\right\|+c_{n}^{(1)}\left\|u_{n}-r\right\|+c_{n}^{(1)}\left\|v_{n}^{(1)}-r\right\| \\
& \leq\left(1+b_{n}^{(1)}+c_{n}^{(1)}\right)\left\|u_{n}-r\right\|+b_{n}^{(1)} L\left\|u_{n}-r\right\|+c_{n}^{(1)}\left\|v_{n}^{(1)}-r\right\| \\
& =\left[1+(1+L) b_{n}^{(1)}+c_{n}^{(1)}\right]\left\|u_{n}-r\right\|+c_{n}^{(1)}\left\|v_{n}^{(1)}-r\right\| .
\end{aligned}
$$

Substituting (3.15) in (3.14), we have

$$
\begin{aligned}
& \left\|u_{n+1}-u_{n}^{(m-1)}\right\| \\
& \leq\left[(1+L) b_{n}^{(m)}+(1+L)\left(1+L b_{n}^{(m)}\right) b_{n}^{(m-1)}+L(1+L)\left(1+L b_{n}^{(m)}\right) b_{n}^{(m-1)} b_{n}^{(m-2)}\right. \\
& +L^{2}\left(1+L b_{n}^{(m)}\right) b_{n}^{(m-1)} b_{n}^{(m-2)} b_{n}^{(m-3)}+\cdots+L^{m-3}\left(1+L b_{n}^{(m)}\right) b_{n}^{(m-1)} b_{n}^{(m-2)} b_{n}^{(m-3)} \cdots b_{n}^{(2)} \\
& +\left[c_{n}^{(m)}+\left(1+L b_{n}^{(m)}\right) c_{n}^{(m-1)}+L\left(1+L b_{n}^{(m)}\right) b_{n}^{(m-1)} c_{n}^{(m-2)}\right. \\
& +L^{2}\left(1+L b_{n}^{(m)}\right) b_{n}^{(m-1)} b_{n}^{(m-2)} c_{n}^{(m-3)} \\
& \left.\left.+\cdots+L^{m-3}\left(1+L b_{n}^{(m)}\right) b_{n}^{(m-1)} b_{n}^{(m-2)} \cdots b_{n}^{(2)} c_{n}^{(3)}\right]\right]\left\|u_{n}-r\right\| \\
& +L^{m-2}\left(1+L b_{n}^{(m)}\right) b_{n}^{(m-1)} b_{n}^{(m-2)} b_{n}^{(m-3)} \cdots b_{n}^{(2)}\left[\left(1+(1+L) b_{n}^{(1)}+c_{n}^{(1)}\right)\left\|u_{n}-r\right\|\right. \\
& \left.+c_{n}^{(1)}\left\|v_{n}^{(1)}-r\right\|\right]+c_{n}^{(m)}\left\|v_{n}^{(m)}-r\right\|+c_{n}^{(m-1)}\left(1+L b_{n}^{(m)}\right)\left\|v_{n}^{(m-1)}-r\right\| \\
& +c_{n}^{(m-2)} L\left(1+L b_{n}^{(m)}\right) b_{n}^{(m-1)}\left\|v_{n}^{(m-2)}-r\right\| \\
& +c_{n}^{(m-3)} L^{2}\left(1+L b_{n}^{(m)}\right) b_{n}^{(m-1)} b_{n}^{(m-2)}\left\|v_{n}^{(m-3)}-r\right\| \\
& +\cdots+c_{n}^{(2)} L^{m-3}\left(1+L b_{n}^{(m)}\right) b_{n}^{(m-1)} b_{n}^{(m-2)} \cdots b_{n}^{(3)}\left\|v_{n}^{(2)}-r\right\| \\
& =\left[(1+L) b_{n}^{(m)}+(1+L)\left(1+L b_{n}^{(m)}\right) b_{n}^{(m-1)}+L(1+L)\left(1+L b_{n}^{(m)}\right) b_{n}^{(m-1)} b_{n}^{(m-2)}\right. \\
& +L^{2}(1+L)\left(1+L b_{n}^{(m)}\right) b_{n}^{(m-1)} b_{n}^{(m-2)} b_{n}^{(m-3)} \\
& +\cdots+L^{m-3}(1+L)\left(1+L b_{n}^{(m)}\right) b_{n}^{(m-1)} b_{n}^{(m-2)} \cdots b_{n}^{(2)} \\
& +L^{m-2}(1+L)\left(1+L b_{n}^{(m)}\right) b_{n}^{(m-1)} b_{n}^{(m-2)} \cdots b_{n}^{(2)} b_{n}^{(1)} \\
& +\left[c_{n}^{(m)}+\left(1+L b_{n}^{(m)}\right) c_{n}^{(m-1)}+L\left(1+L b_{n}^{(m)}\right) b_{n}^{(m-1)} c_{n}^{(m-2)}\right. \\
& +L^{2}\left(1+L b_{n}^{(m)}\right) b_{n}^{(m-1)} b_{n}^{(m-2)} c_{n}^{(m-3)} \\
& +\cdots+L^{m-3}\left(1+L b_{n}^{(m)}\right) b_{n}^{(m-1)} b_{n}^{(m-2)} \cdots b_{n}^{(2)} c_{n}^{(2)}
\end{aligned}
$$




$$
\begin{aligned}
& \left.\left.+L^{m-2}\left(1+L b_{n}^{(m)}\right) b_{n}^{(m-1)} b_{n}^{(m-2)} \cdots b_{n}^{(2)} c_{n}^{(1)}\right]\right]\left\|u_{n}-r\right\| \\
& +c_{n}^{(m)}\left\|v_{n}^{(m)}-r\right\|+c_{n}^{(m-1)}\left(1+L b_{n}^{(m)}\right)\left\|v_{n}^{(m-1)}-r\right\| \\
& +c_{n}^{(m-2)} L\left(1+L b_{n}^{(m)}\right) b_{n}^{(m-1)}\left\|v_{n}^{(m-2)}-r\right\| \\
& +c_{n}^{(m-3)} L^{2}\left(1+L b_{n}^{(m)}\right) b_{n}^{(m-1)} b_{n}^{(m-2)}\left\|v_{n}^{(m-3)}-r\right\| \\
& +\cdots+c_{n}^{(2)} L^{m-3}\left(1+L b_{n}^{(m)}\right) b_{n}^{(m-1)} b_{n}^{(m-2)} \cdots b_{n}^{(3)}\left\|v_{n}^{(2)}-r\right\| \\
& +c_{n}^{(1)} L^{m-2}\left(1+L b_{n}^{(m)}\right) b_{n}^{(m-1)} b_{n}^{(m-2)} \cdots b_{n}^{(2)}\left\|v_{n}^{(1)}-r\right\| .
\end{aligned}
$$

Substituting (3.16) in (3.6), we have

$$
\begin{aligned}
& \left\|u_{n+1}-r\right\| \\
& \leq\left(1-\lambda b_{n}^{(m)}+\lambda^{-1} c_{n}^{(m)}\right)\left\|u_{n}-r\right\| \\
& +\lambda^{-1} L b_{n}^{(m)}\left[\left[(1+L) b_{n}^{(m)}+(1+L)\left(1+L b_{n}^{(m)}\right) b_{n}^{(m-1)}\right.\right. \\
& +L(1+L)\left(1+L b_{n}^{(m)}\right) b_{n}^{(m-1)} b_{n}^{(m-2)} \\
& +L^{2}(1+L)\left(1+L b_{n}^{(m)}\right) b_{n}^{(m-1)} b_{n}^{(m-2)} b_{n}^{(m-3)} \\
& +\cdots+L^{m-3}(1+L)\left(1+L b_{n}^{(m)}\right) b_{n}^{(m-1)} b_{n}^{(m-2)} \cdots b_{n}^{(2)} \\
& +L^{m-2}(1+L)\left(1+L b_{n}^{(m)}\right) b_{n}^{(m-1)} b_{n}^{(m-2)} \cdots b_{n}^{(2)} b_{n}^{(1)} \\
& +\left[c_{n}^{(m)}+\left(1+L b_{n}^{(m)}\right) c_{n}^{(m-1)}+L\left(1+L b_{n}^{(m)}\right) b_{n}^{(m-1)} c_{n}^{(m-2)}\right. \\
& +L^{2}\left(1+L b_{n}^{(m)}\right) b_{n}^{(m-1)} b_{n}^{(m-2)} c_{n}^{(m-3)}+\cdots+L^{m-3}\left(1+L b_{n}^{(m)}\right) b_{n}^{(m-1)} b_{n}^{(m-2)} \cdots b_{n}^{(2)} c_{n}^{(2)} \\
& \left.\left.+L^{m-2}\left(1+L b_{n}^{(m)}\right) b_{n}^{(m-1)} b_{n}^{(m-2)} \cdots b_{n}^{(2)} c_{n}^{(1)}\right]\right]\left\|u_{n}-r\right\| \\
& +c_{n}^{(m)}\left\|v_{n}^{(m)}-r\right\|+c_{n}^{(m-1)}\left(1+L b_{n}^{(m)}\right)\left\|v_{n}^{(m-1)}-r\right\| \\
& +c_{n}^{(m-2)} L\left(1+L b_{n}^{(m)}\right) b_{n}^{(m-1)}\left\|v_{n}^{(m-2)}-r\right\| \\
& +c_{n}^{(m-3)} L^{2}\left(1+L b_{n}^{(m)}\right) b_{n}^{(m-1)} b_{n}^{(m-2)}\left\|v_{n}^{(m-3)}-r\right\| \\
& +\cdots+c_{n}^{(2)} L^{m-3}\left(1+L b_{n}^{(m)}\right) b_{n}^{(m-1)} b_{n}^{(m-2)} \cdots b_{n}^{(3)}\left\|v_{n}^{(2)}-r\right\| \\
& \left.+c_{n}^{(1)} L^{m-2}\left(1+L b_{n}^{(m)}\right) b_{n}^{(m-1)} b_{n}^{(m-2)} \cdots b_{n}^{(2)}\left\|v_{n}^{(1)}-r\right\|\right]+\lambda^{-1} c_{n}^{(m)}\left\|v_{n}^{(m)}-r\right\| \\
& =\left(1-\lambda b_{n}^{(m)}+\lambda^{-1} c_{n}^{(m)}\right)\left\|u_{n}-r\right\| \\
& +\lambda^{-1} L b_{n}^{(m)}\left[(1+L) b_{n}^{(m)}+(1+L)\left(1+L b_{n}^{(m)}\right) b_{n}^{(m-1)}\right. \\
& +L(1+L)\left(1+L b_{n}^{(m)}\right) b_{n}^{(m-1)} b_{n}^{(m-2)} \\
& +L^{2}(1+L)\left(1+L b_{n}^{(m)}\right) b_{n}^{(m-1)} b_{n}^{(m-2)} b_{n}^{(m-3)} \\
& +\cdots+L^{m-3}(1+L)\left(1+L b_{n}^{(m)}\right) b_{n}^{(m-1)} b_{n}^{(m-2)} \cdots b_{n}^{(2)} \\
& +L^{m-2}(1+L)\left(1+L b_{n}^{(m)}\right) b_{n}^{(m-1)} b_{n}^{(m-2)} \cdots b_{n}^{(2)} b_{n}^{(1)} \\
& +\left[c_{n}^{(m)}+\left(1+L b_{n}^{(m)}\right) c_{n}^{(m-1)}+L\left(1+L b_{n}^{(m)}\right) b_{n}^{(m-1)} c_{n}^{(m-2)}\right. \\
& +L^{2}\left(1+L b_{n}^{(m)}\right) b_{n}^{(m-1)} b_{n}^{(m-2)} c_{n}^{(m-3)} \\
& +\cdots+L^{m-3}\left(1+L b_{n}^{(m)}\right) b_{n}^{(m-1)} b_{n}^{(m-2)} \cdots b_{n}^{(2)} c_{n}^{(2)} \\
& \left.\left.+L^{m-2}\left(1+L b_{n}^{(m)}\right) b_{n}^{(m-1)} b_{n}^{(m-2)} \cdots b_{n}^{(2)} c_{n}^{(1)}\right]\right]\left\|u_{n}-r\right\|
\end{aligned}
$$




$$
\begin{aligned}
& +c_{n}^{(m)} \lambda^{-1}\left(1+L b_{n}^{(m)}\right)\left\|v_{n}^{(m)}-r\right\|+c_{n}^{(m-1)} \lambda^{-1} L\left(1+L b_{n}^{(m)}\right) b_{n}^{(m)}\left\|v_{n}^{(m-1)}-r\right\| \\
& +c_{n}^{(m-2)} \lambda^{-1} L^{2}\left(1+L b_{n}^{(m)}\right) b_{n}^{(m)} b_{n}^{(m-1)}\left\|v_{n}^{(m-2)}-r\right\| \\
& +\cdots+c_{n}^{(2)} \lambda^{-1} L^{m-2}\left(1+L b_{n}^{(m)}\right) b_{n}^{(m)} b_{n}^{(m-1)} b_{n}^{(m-2)} \cdots b_{n}^{(3)}\left\|v_{n}^{(2)}-r\right\| \\
& +c_{n}^{(1)} \lambda^{-1} L^{m-1}\left(1+L b_{n}^{(m)}\right) b_{n}^{(m)} b_{n}^{(m-1)} b_{n}^{(m-2)} b_{n}^{(m-3)} \cdots b_{n}^{(2)}\left\|v_{n}^{(1)}-r\right\| \\
& \leq\left[1-b_{n}^{(m)}\left[\lambda-\lambda^{-1} L\left[(1+L) b_{n}^{(m)}+(1+L)^{2} b_{n}^{(m-1)}+L(1+L)^{2} b_{n}^{(m-1)} b_{n}^{(m-2)}\right.\right.\right. \\
& +L^{2}(1+L)^{2} b_{n}^{(m-1)} b_{n}^{(m-2)} b_{n}^{(m-3)}+\cdots+L^{m-3}(1+L)^{2} b_{n}^{(m-1)} b_{n}^{(m-2)} \cdots b_{n}^{(2)} \\
& +L^{m-2}(1+L)^{2} b_{n}^{(m-1)} b_{n}^{(m-2)} \cdots b_{n}^{(2)} b_{n}^{(1)} \\
& +c_{n}^{(m)}+(1+L) c_{n}^{(m-1)}+L(1+L) b_{n}^{(m-1)} c_{n}^{(m-2)} \\
& +L^{2}(1+L) b_{n}^{(m-1)} b_{n}^{(m-2)} c_{n}^{(m-3)}+\cdots+L^{m-3}(1+L) b_{n}^{(m-1)} b_{n}^{(m-2)} \cdots b_{n}^{(3)} c_{n}^{(2)} \\
& \left.\left.\left.+L^{m-2}(1+L) b_{n}^{(m-1)} b_{n}^{(m-2)} b_{n}^{(m-3)} \cdots b_{n}^{(2)} c_{n}^{(1)}\right]-\lambda^{-1} \delta_{n}\right]\right]\left\|u_{n}-r\right\| \\
& +\lambda^{-1}\left(1+L b_{n}^{(m)}\right)\left[c_{n}^{(m)}\left\|v_{n}^{(m)}-q\right\|+c_{n}^{(m-1)} L b_{n}^{(m)}\left\|v_{n}^{(m-1)}-r\right\|\right. \\
& +c_{n}^{(m-2)} L^{2} b_{n}^{(m)} b_{n}^{(m-1)}\left\|v_{n}^{(m-2)}-r\right\|+c_{n}^{(m-3)} L^{3} b_{n}^{(m)} b_{n}^{(m-1)} b_{n}^{(m-2)}\left\|v_{n}^{(m-3)}-r\right\| \\
& +\cdots+c_{n}^{(2)} L^{m-2} b_{n}^{(m)} b_{n}^{(m-1)} b_{n}^{(m-2)} \cdots b_{n}^{(3)}\left\|v_{n}^{(2)}-r\right\| \\
& \left.+c_{n}^{(1)} L^{m-1} b_{n}^{(m)} b_{n}^{(m-1)} b_{n}^{(m-2)} \cdots b_{n}^{(2)}\left\|v_{n}^{(1)}-r\right\|\right] \\
& \leq\left(1-\theta b_{n}^{(m)}\right)\left\|u_{n}-r\right\|+\lambda^{-1}(1+L)\left[c_{n}^{(m)}\left\|v_{n}^{(m)}-q\right\|+c_{n}^{(m-1)} L b_{n}^{(m)}\left\|v_{n}^{(m-1)}-r\right\|\right. \\
& +c_{n}^{(m-2)} L^{2} b_{n}^{(m)} b_{n}^{(m-1)}\left\|v_{n}^{(m-2)}-r\right\|+c_{n}^{(m-3)} L^{3} b_{n}^{(m)} b_{n}^{(m-1)} b_{n}^{(m-2)}\left\|v_{n}^{(m-3)}-r\right\| \\
& +\cdots+c_{n}^{(2)} L^{m-2} b_{n}^{(m)} b_{n}^{(m-1)} b_{n}^{(m-2)} \cdots b_{n}^{(3)}\left\|v_{n}^{(2)}-r\right\| \\
& \left.+c_{n}^{(1)} L^{m-1} b_{n}^{(m)} b_{n}^{(m-1)} b_{n}^{(m-2)} \cdots b_{n}^{(2)}\left\|v_{n}^{(1)}-r\right\|\right] \\
& \leq\left(1-\theta b_{n}^{(m)}\right)\left\|u_{n}-r\right\|+\delta_{n} \lambda^{-1}(1+L) b_{n}^{(m)} A\left[1+L b_{n}^{(m)} b_{n}^{(m-1)}+L^{2} b_{n}^{(m)} b_{n}^{(m-1)} b_{n}^{(m-2)}\right. \\
& \left.+L^{3} b_{n}^{(m)} b_{n}^{(m-1)} b_{n}^{(m-2)} b_{n}^{(m-3)}+\cdots+L^{m-2} b_{n}^{(m)} b_{n}^{(m-1)} b_{n}^{(m-2)} \cdots b_{n}^{(3)}\right] \\
& \left.+L^{m-1} b_{n}^{(m)} b_{n}^{(m-1)} b_{n}^{(m-2)} \cdots b_{n}^{(2)}\right] \text {. }
\end{aligned}
$$

Now, if we put

$$
\begin{aligned}
& \alpha_{n}=\left\|u_{n}-r\right\|, \\
& \omega_{n}= \theta b_{n}^{(m)}, \\
& \beta_{n}= \theta^{-1} \delta_{n} \lambda^{-1}(1+L) b_{n}^{(m)} A\left[1+L b_{n}^{(m)} b_{n}^{(m-1)}\right. \\
&+L^{2} b_{n}^{(m)} b_{n}^{(m-1)} b_{n}^{(m-2)}+L^{3} b_{n}^{(m)} b_{n}^{(m-1)} b_{n}^{(m-2)} b_{n}^{(m-3)} \\
&\left.+\cdots+L^{m-2} b_{n}^{(m)} b_{n}^{(m-1)} b_{n}^{(m-2)} \cdots b_{n}^{(3)}+L^{m-1} b_{n}^{(m)} b_{n}^{(m-1)} b_{n}^{(m-2)} \cdots b_{n}^{(2)}\right], \\
& \gamma_{n}=0,
\end{aligned}
$$

in (3.17), then, by condition (3), we observe that

$$
\alpha_{n+1} \leq\left(1-\omega_{n}\right) \alpha_{n}+\omega_{n} \beta_{n}+\gamma_{n}, \quad n \geq 0,
$$

with $\left\{\omega_{n}\right\}_{n=0}^{\infty} \subset[0,1], \sum_{n=0}^{\infty} \omega_{n}=\infty, \sum_{n=0}^{\infty} \gamma_{n}<\infty$ and $\lim _{n \rightarrow \infty} \beta_{n}=0$. 
Hence, from Lemma 2.4, we have

$$
\lim _{n \rightarrow \infty} \alpha_{n}=0
$$

That is $\lim _{n \rightarrow \infty}\left\|u_{n}-r\right\|=0$.

This ensures that the sequence $\left\{u_{n}\right\}_{n=0}^{\infty}$ of the MMNIPE given by (1.5) converges strongly to the common fixed $r$ of $T$ and $S$.

(ii) From the first equation of (3.1), we get

$$
\begin{aligned}
x_{n}=a_{n}^{(m)} w_{n}+b_{n}^{(m)} T x_{n}^{(m-1)}+c_{n}^{(m)} v_{n}^{(m)} & \\
\Rightarrow \quad\left(1-b_{n}^{(m)}\right) w_{n}= & \left(1-(1-\lambda) b_{n}^{(m)}\right) x_{n}+b_{n}^{(m)}(I-T-\lambda I) x_{n} \\
& +b_{n}^{(m)}\left(T x_{n}-T x_{n}^{(m-1)}\right)-c_{n}^{(m)}\left(v_{n}^{(m)}-w_{n}\right) .
\end{aligned}
$$

Now, for all $n \geq 0$ combining (3.5) and (3.18), we obtain

$$
\begin{aligned}
\left(1-b_{n}^{(m)}\right)\left\|w_{n}-r\right\| \geq & \left\|\left(1-(1-\lambda) b_{n}^{(m)}\right)\left(x_{n}-r\right)+b_{n}^{(m)}(I-T-\lambda I)\left(x_{n}-r\right)\right\| \\
& -b_{n}^{(m)}\left\|T x_{n}-T x_{n}^{(m-1)}\right\|-c_{n}^{(m)}\left\|v_{n}^{(m)}-w_{n}\right\| \\
= & \left(1-(1-\lambda) b_{n}^{(m)}\right)\left\|x_{n}-r+\frac{b_{n}^{(m)}}{1-(1-\lambda) b_{n}^{(m)}}(I-T-\lambda I)\left(x_{n}-r\right)\right\| \\
& -b_{n}^{(m)}\left\|T x_{n}-T x_{n}^{(m-1)}\right\|-c_{n}^{(m)}\left\|v_{n}^{(m)}-w_{n}\right\| \\
\geq & \left(1-(1-\lambda) b_{n}^{(m)}\right)\left\|x_{n}-r\right\|-b_{n}^{(m)}\left\|T x_{n}-T x_{n}^{(m-1)}\right\|-c_{n}^{(m)}\left\|v_{n}^{(m)}-w_{n}\right\| \\
\Rightarrow x_{n}-r \| \leq & \frac{\left(1-b_{n}^{(m)}\right)}{\left(1-(1-\lambda) b_{n}^{(m)}\right)}\left\|w_{n}-r\right\|+\frac{b_{n}^{(m)}}{\left(1-(1-\lambda) b_{n}^{(m)}\right)}\left\|T x_{n}-T x_{n}^{(m-1)}\right\| \\
& +\frac{\left(1-(1-\lambda) b_{n}^{(m)}\right)}{(1-(m)}\left\|v_{n}^{(m)}-w_{n}\right\| \\
\leq & \left(1-\lambda b_{n}^{(m)}\right)\left\|w_{n}-r\right\|+\lambda^{-1} L b_{n}^{(m)}\left\|x_{n}-x_{n}^{(m-1)}\right\|+\lambda^{-1} c_{n}^{(m)}\left\|v_{n}^{(m)}-w_{n}\right\| \\
\leq & \left(1-\lambda b_{n}^{(m)}\right)\left\|w_{n}-r\right\|+\lambda^{-1} L b_{n}^{(m)}\left\|x_{n}-x_{n}^{(m-1)}\right\| \\
& +\lambda^{-1} c_{n}^{(m)}\left(\left\|v_{n}^{(m)}-r\right\|+\left\|w_{n}-r\right\|\right) \\
= & \left(1-\lambda b_{n}^{(m)}+\lambda^{-1} c_{n}^{(m)}\right)\left\|w_{n}-r\right\|+\lambda^{-1} L b_{n}^{(m)}\left\|x_{n}-x_{n}^{(m-1)}\right\| \\
& +\lambda^{-1} c_{n}^{(m)}\left\|v_{n}^{(m)}-r\right\| .
\end{aligned}
$$

But, applying the second equation of (3.1), we have

$$
\begin{aligned}
\| x_{n}- & x_{n}^{(m-1)} \| \\
\leq & \left\|b_{n}^{(m)}\left(T x_{n}^{(m-1)}-w_{n}\right)+c_{n}^{(m)}\left(v_{n}^{(m)}-w_{n}\right)\right\| \\
& +\left\|b_{n}^{(m-1)}\left(w_{n}-T x_{n}^{(m-2)}\right)-c_{n}^{(m-1)}\left(v_{n}^{(m-1)}-w_{n}\right)\right\| \\
\leq & b_{n}^{(m)}\left\|w_{n}-T x_{n}^{(m-1)}\right\|+c_{n}^{(m)}\left\|v_{n}^{(m)}-w_{n}\right\|+b_{n}^{(m-1)}\left\|w_{n}-T x_{n}^{(m-2)}\right\| \\
& +c_{n}^{(m-1)}\left\|v_{n}^{(m-1)}-w_{n}\right\| \\
\leq & b_{n}^{(m)}\left(\left\|w_{n}-r\right\|+\left\|r-T x_{n}^{(m-1)}\right\|\right)+c_{n}^{(m)}\left(\left\|v_{n}^{(m)}-r\right\|+\left\|w_{n}-r\right\|\right)
\end{aligned}
$$




$$
\begin{aligned}
& +b_{n}^{(m-1)}\left(\left\|w_{n}-r\right\|+\left\|r-T x_{n}^{(m-2)}\right\|\right)+c_{n}^{(m-1)}\left(\left\|v_{n}^{(m-1)}-r\right\|+\left\|w_{n}-r\right\|\right) \\
\leq & b_{n}^{(m)}\left(\left\|w_{n}-r\right\|+L\left\|x_{n}^{(m-1)}-r\right\|\right)+c_{n}^{(m)}\left(\left\|v_{n}^{(m)}-r\right\|+\left\|w_{n}-r\right\|\right) \\
& +b_{n}^{(m-1)}\left(\left\|w_{n}-r\right\|+L\left\|x_{n}^{(m-2)}-r\right\|\right)+c_{n}^{(m-1)}\left(\left\|v_{n}^{(m-1)}-r\right\|+\left\|w_{n}-r\right\|\right) \\
\leq & {\left[b_{n}^{(m)}+b_{n}^{(m-1)}+c_{n}^{(m)}+c_{n}^{(m-1)}\right]\left\|w_{n}-r\right\|+L b_{n}^{(m)}\left\|x_{n}^{(m-1)}-r\right\| } \\
& +L b_{n}^{(m-1)}\left\|x_{n}^{(m-2)}-r\right\|+c_{n}^{(m)}\left\|v_{n}^{(m)}-r\right\|+c_{n}^{(m-1)}\left\|v_{n}^{(m-1)}-r\right\| .
\end{aligned}
$$

But after a simple calculation we get

$$
\begin{aligned}
& \left\|x_{n}^{(m-1)}-r\right\| \\
& \leq \quad\left\|w_{n}-r\right\|+L b_{n}^{(m-1)}\left\|x_{n}^{(m-2)}-r\right\|+b_{n}^{(m-1)}\left\|w_{n}-r\right\|+c_{n}^{(m-1)}\left\|v_{n}^{(m-1)}-r\right\| \\
& \quad+c_{n}^{(m-1)}\left\|w_{n}-r\right\| \\
& =\left[1+b_{n}^{(m-1)}+c_{n}^{(m-1)}\right]\left\|w_{n}-r\right\|+L b_{n}^{(m-1)}\left\|x_{n}^{(m-2)}-r\right\|+c_{n}^{(m-1)}\left\|v_{n}^{(m-1)}-r\right\| .
\end{aligned}
$$

Substituting (3.21) in (3.20), we have

$$
\begin{aligned}
\| x_{n}- & x_{n}^{(m-1)} \| \\
\leq & {\left[b_{n}^{(m)}+b_{n}^{(m-1)}+c_{n}^{(m)}+c_{n}^{(m-1)}\right]\left\|w_{n}-r\right\| } \\
& +L b_{n}^{(m)}\left[\left[1+b_{n}^{(m-1)}+c_{n}^{(m-1)}\right]\left\|w_{n}-r\right\|+L b_{n}^{(m-1)}\left\|x_{n}^{(m-2)}-r\right\|+c_{n}^{(m-1)}\left\|v_{n}^{(m-1)}-r\right\|\right] \\
& +L b_{n}^{(m-1)}\left\|x_{n}^{(m-2)}-r\right\|+c_{n}^{(m)}\left\|v_{n}^{(m)}-r\right\|+c_{n}^{(m-1)}\left\|v_{n}^{(m-1)}-r\right\| \\
= & \left.(1+L) b_{n}^{(m)}+\left(1+L b_{n}^{(m)}\right) b_{n}^{(m-1)}+c_{n}^{(m)}+\left(1+L b_{n}^{(m)}\right) c_{n}^{(m-1)}\right]\left\|w_{n}-r\right\| \\
& +L b_{n}^{(m-1)}\left(1+L b_{n}^{(m)}\right)\left\|x_{n}^{(m-2)}-r\right\|+c_{n}^{(m)}\left\|v_{n}^{(m)}-r\right\| \\
& +c_{n}^{(m-1)}\left(1+L b_{n}^{(m)}\right)\left\|v_{n}^{(m-1)}-r\right\| .
\end{aligned}
$$

Now, from the third equation of (3.1), we get

$$
\begin{aligned}
\left\|x_{n}^{(m-2)}-r\right\| & \\
\leq & \left\|w_{n}-r\right\|+L b_{n}^{(m-2)}\left\|x_{n}^{(m-3)}-r\right\|+b_{n}^{(m-2)}\left\|w_{n}-r\right\|+c_{n}^{(m-2)}\left\|v_{n}^{(m-2)}-r\right\| \\
& \quad+c_{n}^{(m-2)}\left\|w_{n}-r\right\| \\
= & {\left[1+b_{n}^{(m-2)}+c_{n}^{(m-2)}\right]\left\|w_{n}-r\right\|+L b_{n}^{(m-2)}\left\|x_{n}^{(m-3)}-r\right\|+c_{n}^{(m-2)}\left\|v_{n}^{(m-2)}-r\right\| . }
\end{aligned}
$$

Substituting (3.23) in (3.22), we have

$$
\begin{aligned}
\| x_{n}- & x_{n}^{(m-1)} \| \\
\leq & {\left[(1+L) b_{n}^{(m)}+\left(1+L b_{n}^{(m)}\right) b_{n}^{(m-1)}+c_{n}^{(m)}+\left(1+L b_{n}^{(m)}\right) c_{n}^{(m-1)}\right]\left\|w_{n}-r\right\| } \\
& +L b_{n}^{(m-1)}\left(1+L b_{n}^{(m)}\right)\left[\left[1+b_{n}^{(m-2)}+c_{n}^{(m-2)}\right]\left\|w_{n}-r\right\|+L b_{n}^{(m-2)}\left\|x_{n}^{(m-3)}-r\right\|\right. \\
& \left.+c_{n}^{(m-2)}\left\|v_{n}^{(m-2)}-r\right\|\right]+c_{n}^{(m)}\left\|v_{n}^{(m)}-r\right\|+c_{n}^{(m-1)}\left(1+L b_{n}^{(m)}\right)\left\|v_{n}^{(m-1)}-r\right\| \\
= & (1+L) b_{n}^{(m)}+(1+L)\left(1+L b_{n}^{(m)}\right) b_{n}^{(m-1)}+L\left(1+L b_{n}^{(m)}\right) b_{n}^{(m-1)} b_{n}^{(m-2)}+c_{n}^{(m)} \\
& \left.+\left(1+L b_{n}^{(m)}\right) c_{n}^{(m-1)}+L\left(1+L b_{n}^{(m)}\right) b_{n}^{(m-1)} c_{n}^{(m-2)}\right]\left\|w_{n}-r\right\|
\end{aligned}
$$




$$
\begin{aligned}
& +L^{2}\left(1+L b_{n}^{(m)}\right) b_{n}^{(m-1)} b_{n}^{(m-2)}\left\|x_{n}^{(m-3)}-r\right\|+c_{n}^{(m)}\left\|v_{n}^{(m)}-r\right\| \\
& +\left(1+L b_{n}^{(m)}\right) c_{n}^{(m-1)}\left\|v_{n}^{(m-1)}-r\right\|+L\left(1+L b_{n}^{(m)}\right) b_{n}^{(m-1)} c_{n}^{(m-2)}\left\|v_{n}^{(m-2)}-r\right\| .
\end{aligned}
$$

Continuing the above procedure up to second iterative step of (3.1), we obtain

$$
\begin{aligned}
\| x_{n}- & x_{n}^{(m-1)} \| \\
\leq & {\left[(1+L) b_{n}^{(m)}+(1+L)\left(1+L b_{n}^{(m)}\right) b_{n}^{(m-1)}+L(1+L)\left(1+L b_{n}^{(m)}\right) b_{n}^{(m-1)} b_{n}^{(m-2)}\right.} \\
& +L^{2}\left(1+L b_{n}^{(m)}\right) b_{n}^{(m-1)} b_{n}^{(m-2)} b_{n}^{(m-3)}+\cdots+L^{m-3}\left(1+L b_{n}^{(m)}\right) b_{n}^{(m-1)} b_{n}^{(m-2)} b_{n}^{(m-3)} \cdots b_{n}^{(2)} \\
& +\left[c_{n}^{(m)}+\left(1+L b_{n}^{(m)}\right) c_{n}^{(m-1)}+L\left(1+L b_{n}^{(m)}\right) b_{n}^{(m-1)} c_{n}^{(m-2)}\right. \\
& +L^{2}\left(1+L b_{n}^{(m)}\right) b_{n}^{(m-1)} b_{n}^{(m-2)} c_{n}^{(m-3)} \\
& \left.\left.+\cdots+L^{m-3}\left(1+L b_{n}^{(m)}\right) b_{n}^{(m-1)} b_{n}^{(m-2)} \cdots b_{n}^{(2)} c_{n}^{(3)}\right]\right]\left\|w_{n}-r\right\| \\
& +L^{m-2}\left(1+L b_{n}^{(m)}\right) b_{n}^{(m-1)} b_{n}^{(m-2)} b_{n}^{(m-3)} \cdots b_{n}^{(2)}\left\|x_{n}^{(1)}-r\right\|+\left[c_{n}^{(m)}\left\|v_{n}^{(m)}-r\right\|\right. \\
& +\left(1+L b_{n}^{(m)}\right) c_{n}^{(m-1)}\left\|v_{n}^{(m-1)}-r\right\|+L\left(1+L b_{n}^{(m)}\right) b_{n}^{(m-1)} c_{n}^{(m-2)}\left\|v_{n}^{(m-2)}-r\right\| \\
& +L^{2}\left(1+L b_{n}^{(m)}\right) b_{n}^{(m-1)} b_{n}^{(m-2)} c_{n}^{(m-3)}\left\|v_{n}^{(m-3)}-r\right\| \\
& \left.+\cdots+L^{m-3}\left(1+L b_{n}^{(m)}\right) b_{n}^{(m-1)} b_{n}^{(m-2)} \cdots b_{n}^{(3)} c_{n}^{(2)}\left\|v_{n}^{(2)}-r\right\|\right] .
\end{aligned}
$$

Now, from the last equation of (3.1), we have

$$
\begin{aligned}
\left\|x_{n}^{(1)}-r\right\| & =\left\|a_{n}^{(1)} w_{n}+b_{n}^{(1)} S w_{n}+c_{n}^{(1)} v_{n}^{(1)}-r\right\| \\
& \leq\left\|w_{n}-r\right\|+b_{n}^{(1)}\left\|S w_{n}-r\right\|+b_{n}^{(1)}\left\|w_{n}-r\right\|+c_{n}^{(1)}\left\|w_{n}-r\right\|+c_{n}^{(1)}\left\|v_{n}^{(1)}-r\right\| \\
& =\left(1+b_{n}^{(1)}+c_{n}^{(1)}\right)\left\|w_{n}-r\right\|+b_{n}^{(1)}\left\|S w_{n}-r\right\|+c_{n}^{(1)}\left\|v_{n}^{(1)}-r\right\| \\
& \leq\left(1+b_{n}^{(1)}+c_{n}^{(1)}\right)\left\|w_{n}-r\right\|+b_{n}^{(1)} L\left\|w_{n}-r\right\|+c_{n}^{(1)}\left\|v_{n}^{(1)}-r\right\| \\
& =\left(1+(1+L) b_{n}^{(1)}+c_{n}^{(1)}\right)\left\|w_{n}-r\right\|+c_{n}^{(1)}\left\|v_{n}^{(1)}-r\right\| .
\end{aligned}
$$

Combining (3.24) and (3.25), we obtain

$$
\begin{aligned}
\| x_{n}- & x_{n}^{(m-1)} \| \\
\leq & (1+L) b_{n}^{(m)}+(1+L)\left(1+L b_{n}^{(m)}\right) b_{n}^{(m-1)}+L(1+L)\left(1+L b_{n}^{(m)}\right) b_{n}^{(m-1)} b_{n}^{(m-2)} \\
& +L^{2}(1+L)\left(1+L b_{n}^{(m)}\right) b_{n}^{(m-1)} b_{n}^{(m-2)} b_{n}^{(m-3)} \\
& +\cdots+L^{m-3}(1+L)\left(1+L b_{n}^{(m)}\right) b_{n}^{(m-1)} b_{n}^{(m-2)} b_{n}^{(m-3)} \cdots b_{n}^{(2)} \\
& +L^{m-2}(1+L)\left(1+L b_{n}^{(m)}\right) b_{n}^{(m-1)} b_{n}^{(m-2)} b_{n}^{(m-3)} \cdots b_{n}^{(2)} b_{n}^{(1)} \\
& +\left[c_{n}^{(m)}+\left(1+L b_{n}^{(m)}\right) c_{n}^{(m-1)}+L\left(1+L b_{n}^{(m)}\right) b_{n}^{(m-1)} c_{n}^{(m-2)}\right. \\
& +L^{2}\left(1+L b_{n}^{(m)}\right) b_{n}^{(m-1)} b_{n}^{(m-2)} c_{n}^{(m-3)}+\cdots+L^{m-3}\left(1+L b_{n}^{(m)}\right) b_{n}^{(m-1)} b_{n}^{(m-2)} \cdots b_{n}^{(3)} c_{n}^{(2)} \\
& \left.\left.+L^{m-2}\left(1+L b_{n}^{(m)}\right) b_{n}^{(m-1)} b_{n}^{(m-2)} b_{n}^{(m-3)} \cdots b_{n}^{(2)} c_{n}^{(1)}\right]\right]\left\|w_{n}-r\right\| \\
& +\left[c_{n}^{(m)}\left\|v_{n}^{(m)}-r\right\|+\left(1+L b_{n}^{(m)}\right) c_{n}^{(m-1)}\left\|v_{n}^{(m-1)}-r\right\|\right. \\
& +L\left(1+L b_{n}^{(m)}\right) b_{n}^{(m-1)} c_{n}^{(m-2)}\left\|v_{n}^{(m-2)}-r\right\|
\end{aligned}
$$




$$
\begin{aligned}
& +L^{2}\left(1+L b_{n}^{(m)}\right) b_{n}^{(m-1)} b_{n}^{(m-2)} c_{n}^{(m-3)}\left\|v_{n}^{(m-3)}-r\right\| \\
& +\cdots+L^{m-3}\left(1+L b_{n}^{(m)}\right) b_{n}^{(m-1)} b_{n}^{(m-2)} \cdots b_{n}^{(3)} c_{n}^{(2)}\left\|v_{n}^{(2)}-r\right\| \\
& \left.+L^{m-2}\left(1+L b_{n}^{(m)}\right) b_{n}^{(m-1)} b_{n}^{(m-2)} b_{n}^{(m-3)} \cdots b_{n}^{(2)} c_{n}^{(1)}\left\|v_{n}^{(1)}-r\right\|\right]
\end{aligned}
$$

Inserting (3.26) in (3.19), we find

$$
\begin{aligned}
& \left\|x_{n}-r\right\| \\
& \leq\left[1-\lambda b_{n}^{(m)}+\lambda^{-1} c_{n}^{(m)}+\lambda^{-1} L b_{n}^{(m)}\left[(1+L) b_{n}^{(m)}+(1+L)\left(1+L b_{n}^{(m)}\right) b_{n}^{(m-1)}\right.\right. \\
& +L(1+L)\left(1+L b_{n}^{(m)}\right) b_{n}^{(m-1)} b_{n}^{(m-2)}+L^{2}(1+L)\left(1+L b_{n}^{(m)}\right) b_{n}^{(m-1)} b_{n}^{(m-2)} b_{n}^{(m-3)} \\
& +\cdots+L^{m-3}(1+L)\left(1+L b_{n}^{(m)}\right) b_{n}^{(m-1)} b_{n}^{(m-2)} b_{n}^{(m-3)} \cdots b_{n}^{(2)} \\
& +L^{m-2}(1+L)\left(1+L b_{n}^{(m)}\right) b_{n}^{(m-1)} b_{n}^{(m-2)} b_{n}^{(m-3)} \cdots b_{n}^{(2)} b_{n}^{(1)} \\
& +\left[c_{n}^{(m)}+\left(1+L b_{n}^{(m)}\right) c_{n}^{(m-1)}+L\left(1+L b_{n}^{(m)}\right) b_{n}^{(m-1)} c_{n}^{(m-2)}\right. \\
& +L^{2}\left(1+L b_{n}^{(m)}\right) b_{n}^{(m-1)} b_{n}^{(m-2)} c_{n}^{(m-3)}+\cdots+L^{m-3}\left(1+L b_{n}^{(m)}\right) b_{n}^{(m-1)} b_{n}^{(m-2)} \cdots b_{n}^{(3)} c_{n}^{(2)} \\
& \left.\left.\left.+L^{m-2}\left(1+L b_{n}^{(m)}\right) b_{n}^{(m-1)} b_{n}^{(m-2)} b_{n}^{(m-3)} \cdots b_{n}^{(2)} c_{n}^{(1)}\right]\right]\right]\left\|w_{n}-r\right\| \\
& +\lambda^{-1} L b_{n}^{(m)}\left[c_{n}^{(m)}\left\|v_{n}^{(m)}-r\right\|+\left(1+L b_{n}^{(m)}\right) c_{n}^{(m-1)}\left\|v_{n}^{(m-1)}-r\right\|\right. \\
& +L\left(1+L b_{n}^{(m)}\right) b_{n}^{(m-1)} c_{n}^{(m-2)}\left\|v_{n}^{(m-2)}-r\right\| \\
& +L^{2}\left(1+L b_{n}^{(m)}\right) b_{n}^{(m-1)} b_{n}^{(m-2)} c_{n}^{(m-3)}\left\|v_{n}^{(m-3)}-r\right\| \\
& +\cdots+L^{m-3}\left(1+L b_{n}^{(m)}\right) b_{n}^{(m-1)} b_{n}^{(m-2)} \cdots b_{n}^{(3)} c_{n}^{(2)}\left\|v_{n}^{(2)}-r\right\| \\
& \left.+L^{m-2}\left(1+L b_{n}^{(m)}\right) b_{n}^{(m-1)} b_{n}^{(m-2)} b_{n}^{(m-3)} \cdots b_{n}^{(2)} c_{n}^{(1)}\left\|v_{n}^{(1)}-r\right\|\right]+\lambda^{-1} c_{n}^{(m)}\left\|v_{n}^{(m)}-r\right\| \\
& =\left[1-b_{n}^{(m)}\left[\lambda-\lambda^{-1} L\left[(1+L) b_{n}^{(m)}+(1+L)\left(1+L b_{n}^{(m)}\right) b_{n}^{(m-1)}\right.\right.\right. \\
& +L(1+L)\left(1+L b_{n}^{(m)}\right) b_{n}^{(m-1)} b_{n}^{(m-2)}+L^{2}(1+L)\left(1+L b_{n}^{(m)}\right) b_{n}^{(m-1)} b_{n}^{(m-2)} b_{n}^{(m-3)} \\
& +\cdots+L^{m-3}(1+L)\left(1+L b_{n}^{(m)}\right) b_{n}^{(m-1)} b_{n}^{(m-2)} b_{n}^{(m-3)} \cdots b_{n}^{(2)} \\
& +L^{m-2}(1+L)\left(1+L b_{n}^{(m)}\right) b_{n}^{(m-1)} b_{n}^{(m-2)} b_{n}^{(m-3)} \cdots b_{n}^{(2)} b_{n}^{(1)} \\
& +\left[c_{n}^{(m)}+\left(1+L b_{n}^{(m)}\right) c_{n}^{(m-1)}+L\left(1+L b_{n}^{(m)}\right) b_{n}^{(m-1)} c_{n}^{(m-2)}\right. \\
& +L^{2}\left(1+L b_{n}^{(m)}\right) b_{n}^{(m-1)} b_{n}^{(m-2)} c_{n}^{(m-3)}+\cdots+L^{m-3}\left(1+L b_{n}^{(m)}\right) b_{n}^{(m-1)} b_{n}^{(m-2)} \cdots b_{n}^{(3)} c_{n}^{(2)} \\
& \left.\left.\left.\left.+L^{m-2}\left(1+L b_{n}^{(m)}\right) b_{n}^{(m-1)} b_{n}^{(m-2)} b_{n}^{(m-3)} \cdots b_{n}^{(2)} c_{n}^{(1)}\right]\right]-\lambda^{-1} \delta_{n}\right]\right]\left\|w_{n}-r\right\| \\
& +\lambda^{-1}\left(1+L b_{n}^{(m)}\right) c_{n}^{(m)}\left\|v_{n}^{(m)}-r\right\|+\lambda^{-1} L\left(1+L b_{n}^{(m)}\right) b_{n}^{(m)} c_{n}^{(m-1)}\left\|v_{n}^{(m-1)}-r\right\| \\
& +\lambda^{-1} L^{2}\left(1+L b_{n}^{(m)}\right) b_{n}^{(m)} b_{n}^{(m-1)} c_{n}^{(m-2)}\left\|v_{n}^{(m-2)}-r\right\| \\
& +\lambda^{-1} L^{3}\left(1+L b_{n}^{(m)}\right) b_{n}^{(m)} b_{n}^{(m-1)} b_{n}^{(m-2)} c_{n}^{(m-3)}\left\|v_{n}^{(m-3)}-r\right\| \\
& +\cdots+\lambda^{-1} L^{m-2}\left(1+L b_{n}^{(m)}\right) b_{n}^{(m)} b_{n}^{(m-1)} b_{n}^{(m-2)} \cdots b_{n}^{(3)} c_{n}^{(2)}\left\|v_{n}^{(2)}-r\right\| \\
& +\lambda^{-1} L^{m-1}\left(1+L b_{n}^{(m)}\right) b_{n}^{(m)} b_{n}^{(m-1)} b_{n}^{(m-2)} b_{n}^{(m-3)} \cdots b_{n}^{(2)} c_{n}^{(1)}\left\|v_{n}^{(1)}-r\right\| \\
& \leq\left[1-b_{n}^{(m)}\left[\lambda-\lambda^{-1} L\left[(1+L) b_{n}^{(m)}+(1+L)^{2} b_{n}^{(m-1)}\right.\right.\right. \\
& +L(1+L)^{2} b_{n}^{(m-1)} b_{n}^{(m-2)}+L^{2}(1+L)^{3} b_{n}^{(m-1)} b_{n}^{(m-2)} b_{n}^{(m-3)} \\
& +\cdots+L^{m-3}(1+L)^{2} b_{n}^{(m-1)} b_{n}^{(m-2)} b_{n}^{(m-3)} \cdots b_{n}^{(2)}
\end{aligned}
$$




$$
\begin{aligned}
& +L^{m-2}(1+L)^{2} b_{n}^{(m-1)} b_{n}^{(m-2)} b_{n}^{(m-3)} \cdots b_{n}^{(2)} b_{n}^{(1)} \\
& +\left[c_{n}^{(m)}+(1+L) c_{n}^{(m-1)}+L(1+L) b_{n}^{(m-1)} c_{n}^{(m-2)}\right. \\
& +L^{2}(1+L) b_{n}^{(m-1)} b_{n}^{(m-2)} c_{n}^{(m-3)}+\cdots+L^{m-3}(1+L) b_{n}^{(m-1)} b_{n}^{(m-2)} \cdots b_{n}^{(3)} c_{n}^{(2)} \\
& \left.\left.\left.\left.+L^{m-2}(1+L) b_{n}^{(m-1)} b_{n}^{(m-2)} b_{n}^{(m-3)} \cdots b_{n}^{(2)} c_{n}^{(1)}\right]\right]-\lambda^{-1} \delta_{n}\right]\right]\left\|w_{n}-r\right\| \\
& +\lambda^{-1}(1+L) c_{n}^{(m)}\left\|v_{n}^{(m)}-r\right\|+\lambda^{-1} L(1+L) b_{n}^{(m)} c_{n}^{(m-1)}\left\|v_{n}^{(m-1)}-r\right\| \\
& +\lambda^{-1} L^{2}(1+L) b_{n}^{(m)} b_{n}^{(m-1)} c_{n}^{(m-2)}\left\|v_{n}^{(m-2)}-r\right\| \\
& +\lambda^{-1} L^{3}(1+L) b_{n}^{(m)} b_{n}^{(m-1)} b_{n}^{(m-2)} c_{n}^{(m-3)}\left\|v_{n}^{(m-3)}-r\right\| \\
& +\cdots+\lambda L^{m-2}(1+L) b_{n}^{(m)} b_{n}^{(m-1)} b_{n}^{(m-2)} \cdots b_{n}^{(3)} c_{n}^{(2)}\left\|v_{n}^{(2)}-r\right\| \\
& +\lambda^{-1} L^{m-1}(1+L) b_{n}^{(m)} b_{n}^{(m-1)} b_{n}^{(m-2)} b_{n}^{(m-3)} \cdots b_{n}^{(2)} c_{n}^{(1)}\left\|v_{n}^{(1)}-r\right\| \\
& \leq\left(1-\theta b_{n}^{(m)}\right)\left\|w_{n}-r\right\| \\
& +\lambda^{-1}(1+L)\left[c_{n}^{(m)}\left\|v_{n}^{(m)}-r\right\|+L\left[b_{n}^{(m)} c_{n}^{(m-1)}\left\|v_{n}^{(m-1)}-r\right\|\right.\right. \\
& +L\left[b_{n}^{(m)} b_{n}^{(m-1)} c_{n}^{(m-2)}\left\|v_{n}^{(m-2)}-r\right\|+L\left[b_{n}^{(m)} b_{n}^{(m-1)} b_{n}^{(m-2)} c_{n}^{(m-3)}\left\|v_{n}^{(m-3)}-r\right\|\right.\right. \\
& +\cdots+L\left[b_{n}^{(m)} b_{n}^{(m-1)} b_{n}^{(m-2)} \cdots b_{n}^{(3)} c_{n}^{(2)}\left\|v_{n}^{(2)}-r\right\|\right. \\
& \left.\left.\left.\left.+L\left[b_{n}^{(m)} b_{n}^{(m-1)} b_{n}^{(m-2)} b_{n}^{(m-3)} \ldots b_{n}^{(2)} c_{n}^{(1)}\left\|v_{n}^{(1)}-r\right\|\right]\right]\right]\right] \cdots\right] . \\
&
\end{aligned}
$$

Now, using (3.27), we obtain

$$
\begin{aligned}
\| w_{n+1} & -r \| \\
\leq & \left\|w_{n+1}-x_{n}\right\|+\left\|x_{n}-r\right\| \\
\leq & \left(1-\theta b_{n}^{(m)}\right)\left\|w_{n}-r\right\| \\
& +\lambda^{-1}(1+L)\left[c_{n}^{(m)}\left\|v_{n}^{(m)}-r\right\|+L\left[b_{n}^{(m)} c_{n}^{(m-1)}\left\|v_{n}^{(m-1)}-r\right\|\right.\right. \\
\leq & \left(1-\theta b_{n}^{(m)}\right)\left\|w_{n}-r\right\|+\lambda^{-1}(1+L)\left[c_{n}^{(m)} A+L\left[b_{n}^{(m)} c_{n}^{(m-1)} A\right.\right. \\
& +L\left[b_{n}^{(m)} b_{n}^{(m-1)} c_{n}^{(m-2)} A+L\left[b_{n}^{(m)} b_{n}^{(m-1)} b_{n}^{(m-2)} c_{n}^{(m-3)} A\right.\right. \\
& +\cdots+L\left[b_{n}^{(m)} b_{n}^{(m-1)} b_{n}^{(m-2)} \cdots b_{n}^{(3)} c_{n}^{(2)} A\right. \\
& \left.\left.\left.\left.\left.+L\left[b_{n}^{(m)} b_{n}^{(m-1)} b_{n}^{(m-2)} b_{n}^{(m-3)} \cdots b_{n}^{(2)} c_{n}^{(1)} A\right]\right]\right]\right] \cdots\right]\right]+\mu_{n} \\
= & \left(1-\theta b_{n}^{(m)}\right)\left\|w_{n}-r\right\|+\lambda^{-1}(1+L) A b_{n}^{(m)}\left[\delta_{n}+L\left[c_{n}^{(m-1)}\right.\right. \\
& +L\left[b_{n}^{(m-1)} c_{n}^{(m-2)}+L\left[b_{n}^{(m-1)} b_{n}^{(m-2)} c_{n}^{(m-3)}+\cdots+L\left[b_{n}^{(m-1)} b_{n}^{(m-2)} \cdots b_{n}^{(3)} c_{n}^{(2)}\right.\right.\right. \\
& \left.\left.\left.\left.\left.+L\left[b_{n}^{(m-1)} b_{n}^{(m-2)} b_{n}^{(m-3)} \cdots b_{n}^{(2)} c_{n}^{(1)}\right]\right]\right]\right] \cdots\right]\right]+\mu_{n}, \quad n \geq 0 .
\end{aligned}
$$

(iii) If we put

$$
\begin{aligned}
& \alpha_{n}=\left\|w_{n}-r\right\|, \\
& \omega_{n}=\theta b_{n}^{(m)},
\end{aligned}
$$




$$
\begin{aligned}
\beta_{n}= & \theta^{-1} \lambda^{-1}(1+L) A\left[\delta_{n}+L\left[c_{n}^{(m-1)}+L\left[b_{n}^{(m-1)} c_{n}^{(m-2)}+L\left[b_{n}^{(m-1)} b_{n}^{(m-2)} c_{n}^{(m-3)}\right.\right.\right.\right. \\
& \left.\left.\left.\left.\quad+\cdots+L\left[b_{n}^{(m-1)} b_{n}^{(m-2)} \cdots b_{n}^{(3)} c_{n}^{(2)}+L\left[b_{n}^{(m-1)} b_{n}^{(m-2)} b_{n}^{(m-3)} \cdots b_{n}^{(2)} c_{n}^{(1)}\right]\right]\right]\right] \cdots\right]\right], \\
& \gamma_{n}=\mu_{n}, \quad \forall n \geq 0,
\end{aligned}
$$

in (3.28), then we obtain

$$
\alpha_{n+1} \leq\left(1-\omega_{n}\right) \alpha_{n}+\omega_{n} \beta_{n}+\gamma_{n}, \quad n \geq 0
$$

where $\left\{\omega_{n}\right\}_{n=0}^{\infty} \subset[0,1], \sum_{n=0}^{\infty} \omega_{n}=\infty, \sum_{n=0}^{\infty} \gamma_{n}<\infty$ and $\lim _{n \rightarrow \infty} \beta_{n}=0$.

Hence, from Lemma 2.4, we have $\lim _{n \rightarrow \infty} \alpha_{n}=0$.

That is $\lim _{n \rightarrow \infty}\left\|w_{n}-r\right\|=0$. Hence, $\lim _{n \rightarrow \infty} w_{n}=r$ and this ensures that $\left\{u_{n}^{(m)}\right\}_{n=0}^{\infty}$ is almost common-stable on $B$.

(iv) Considering $\lim _{n \rightarrow \infty} w_{n}=r$, we have $\lim _{n \rightarrow \infty} w_{n+1}=r$.

Now, using (3.27), we get

$$
\begin{aligned}
\mu_{n}= & \left\|w_{n+1}-x_{n}\right\| \leq\left\|w_{n+1}-r\right\|+\left\|x_{n}-r\right\| \\
\leq & \left\|w_{n+1}-r\right\|+\left(1-\theta b_{n}^{(m)}\right)\left\|w_{n}-r\right\| \\
& +\lambda^{-1}(1+L) A b_{n}^{(m)}\left[\delta_{n}+L\left[c_{n}^{(m-1)}+L\left[b_{n}^{(m-1)} c_{n}^{(m-2)}+L\left[b_{n}^{(m-1)} b_{n}^{(m-2)} c_{n}^{(m-3)}\right.\right.\right.\right. \\
& \left.\left.\left.\left.+\cdots+L\left[b_{n}^{(m-1)} b_{n}^{(m-2)} \cdots b_{n}^{(3)} c_{n}^{(2)}+L b_{n}^{(m-1)} b_{n}^{(m-2)} b_{n}^{(m-3)} \cdots b_{n}^{(2)} c_{n}^{(1)}\right]\right]\right] \cdots\right]\right] \rightarrow 0,
\end{aligned}
$$

as $n \rightarrow \infty$, this means that $\lim _{n \rightarrow \infty} \mu_{n}=0$.

This completes the proof.

Theorem 3.2 Let $B, X, T, S, \theta,\left\{u_{n}\right\}_{n=0}^{\infty},\left\{x_{n}\right\}_{n=0}^{\infty},\left\{w_{n}\right\}_{n=0}^{\infty},\left\{v_{n}^{(1)}\right\}, \ldots,\left\{v_{n}^{(m)}\right\}$, and $\left\{\mu_{n}\right\}_{n=0}^{\infty}$ be as in Theorem 3.1 and $\left\{a_{n}^{(i)}\right\},\left\{b_{n}^{(i)}\right\},\left\{c_{n}^{(i)}\right\}$ for each $i \in\{1,2, \ldots, m\}$ be any appropriate real sequences in $[0,1]$ satisfying the conditions (1), (3), (4), (5) of Theorem 3.1 with the following property:

$$
\sum_{n=0}^{\infty} c_{n}^{(m)}<\infty
$$

Then the results of Theorem 3.1 hold.

Proof The proof of this theorem is similar to the proof of Theorem 3.1, so here we omit it.

Theorem 3.3 Let $B, X, T, S, \theta,\left\{u_{n}\right\}_{n=0}^{\infty},\left\{x_{n}\right\}_{n=0}^{\infty},\left\{w_{n}\right\}_{n=0}^{\infty},\left\{v_{n}^{(1)}\right\}, \ldots,\left\{v_{n}^{(m)}\right\}$, and $\left\{\mu_{n}\right\}_{n=0}^{\infty}$ be as in Theorem 3.1 and $\left\{a_{n}^{(i)}\right\},\left\{b_{n}^{(i)}\right\},\left\{c_{n}^{(i)}\right\}$ for each $i \in\{1,2, \ldots, m\}$ be any appropriate real sequences in $[0,1]$ satisfying the conditions (1), (3) and (5) of Theorem 3.1 with the following property:

$$
\lim _{n \rightarrow \infty} c_{n}^{(m)}=0, \quad \text { and } \quad b_{n}^{(m)} \geq h>0, \quad \forall n \geq 0
$$

where h is a constant.

Then 
(i) the iterative sequence $\left\{u_{n}\right\}_{n=0}^{\infty}$ given by (1.5) converges strongly to the common fixed $r$ of $T$ and $S$ and the following inequality holds:

$$
\left\|u_{n+1}-r\right\| \leq(1-\theta h)\left\|u_{n}-r\right\|+D, \quad \forall n \geq 0,
$$

where

$$
\begin{aligned}
D= & \lambda^{-1}(1+L)\left[\sup _{n \geq 0}\left\{c_{n}^{(m)}\left\|v_{n}^{(m)}-r\right\|\right\}+L b_{n}^{(m)} \sup _{n \geq 0}\left\{c_{n}^{(m-1)}\left\|v_{n}^{(m-1)}-r\right\|\right\}\right. \\
& +L^{2} b_{n}^{(m)} b_{n}^{(m-1)} \sup _{n \geq 0}\left\{c_{n}^{(m-2)}\left\|v_{n}^{(m-2)}-r\right\|\right\} \\
& +L^{3} b_{n}^{(m)} b_{n}^{(m-1)} b_{n}^{(m-2)} \sup _{n \geq 0}\left\{c_{n}^{(m-3)}\left\|v_{n}^{(m-3)}-r\right\|\right\} \\
& +\cdots+L^{m-2} b_{n}^{(m)} b_{n}^{(m-1)} b_{n}^{(m-2)} \cdots b_{n}^{(3)} \sup _{n \geq 0}\left\{c_{n}^{(2)}\left\|v_{n}^{(2)}-r\right\|\right\} \\
& \left.+L^{m-1} b_{n}^{(m)} b_{n}^{(m-1)} b_{n}^{(m-2)} b_{n}^{(m-3)} \cdots b_{n}^{(2)} \sup _{n \geq 0}\left\{c_{n}^{(1)}\left\|v_{n}^{(1)}-r\right\|\right\}\right]
\end{aligned}
$$

(ii)

$$
\begin{aligned}
& \left\|w_{n+1}-r\right\| \\
& \leq \quad(1-\theta h)\left\|w_{n}-r\right\|+\lambda^{-1}(1+L)\left[c_{n}^{(m)}\left\|v_{n}^{(m)}-r\right\|+L\left[c_{n}^{(m-1)}\left\|v_{n}^{(m-1)}-r\right\|\right.\right. \\
& \quad+L\left[c_{n}^{(m-2)}\left\|v_{n}^{(m-2)}-r\right\|+L\left[c_{n}^{(m-3)}\left\|v_{n}^{(m-3)}-r\right\|\right.\right. \\
& \left.\left.\left.\left.\quad+\cdots+L\left[c_{n}^{(2)}\left\|v_{n}^{(2)}-r\right\|+L\left[c_{n}^{(1)}\left\|v_{n}^{(1)}-r\right\|\right]\right]\right]\right] \cdots\right]\right]+\mu_{n}, \quad \forall n \geq 0,
\end{aligned}
$$

(iii) $\lim _{n \rightarrow \infty} w_{n}=r$, if and only if $\lim _{n \rightarrow \infty} \mu_{n}=0$.

Proof (i) Following the proof of Theorem 3.1, we have

$$
\begin{aligned}
\| u_{n+1} & -r \| \\
\leq & \left(1-\theta b_{n}^{(m)}\right)\left\|u_{n}-r\right\|+\lambda^{-1}(1+L) c_{n}^{(m)}\left\|v_{n}^{(m)}-r\right\|+\lambda^{-1} L(1+L) b_{n}^{(m)} c_{n}^{(m-1)}\left\|v_{n}^{(m-1)}-r\right\| \\
& +\lambda^{-1} L^{2}(1+L) b_{n}^{(m)} b_{n}^{(m-1)} c_{n}^{(m-2)}\left\|v_{n}^{(m-2)}-r\right\| \\
& +\lambda^{-1} L^{3}(1+L) b_{n}^{(m)} b_{n}^{(m-1)} b_{n}^{(m-2)} c_{n}^{(m-3)}\left\|v_{n}^{(m-3)}-r\right\| \\
& +\cdots+\lambda^{-1} L^{m-2}(1+L) b_{n}^{(m)} b_{n}^{(m-1)} b_{n}^{(m-2)} \cdots b_{n}^{(3)} c_{n}^{(2)}\left\|v_{n}^{(2)}-r\right\| \\
& +\lambda^{-1} L^{m-1}(1+L) b_{n}^{(m)} b_{n}^{(m-1)} b_{n}^{(m-2)} b_{n}^{(m-3)} \cdots b_{n}^{(2)} c_{n}^{(1)}\left\|v_{n}^{(1)}-r\right\| \\
\leq & (1-\theta h)\left\|u_{n}-r\right\|+\lambda^{-1}(1+L)\left[c_{n}^{(m)}\left\|v_{n}^{(m)}-r\right\|+L b_{n}^{(m)} c_{n}^{(m-1)}\left\|v_{n}^{(m-1)}-r\right\|\right. \\
& +L^{2} b_{n}^{(m)} b_{n}^{(m-1)} c_{n}^{(m-2)}\left\|v_{n}^{(m-2)}-r\right\|+L^{3} b_{n}^{(m)} b_{n}^{(m-1)} b_{n}^{(m-2)} c_{n}^{(m-3)}\left\|v_{n}^{(m-3)}-r\right\| \\
& +\cdots+L^{m-2} b_{n}^{(m)} b_{n}^{(m-1)} b_{n}^{(m-2)} \cdots b_{n}^{(3)} c_{n}^{(2)}\left\|v_{n}^{(2)}-r\right\| \\
& \left.+L^{m-1} b_{n}^{(m)} b_{n}^{(m-1)} b_{n}^{(m-2)} b_{n}^{(m-3)} \cdots b_{n}^{(2)} c_{n}^{(1)}\left\|v_{n}^{(1)}-r\right\|\right] \\
\leq & (1-\theta h)\left\|u_{n}-r\right\|+\lambda^{-1}(1+L)\left[\sup _{n \geq 0}\left\{c_{n}^{(m)}\left\|v_{n}^{(m)}-r\right\|\right\}+L b_{n}^{(m)} \sup _{n \geq 0}\left\{c_{n}^{(m-1)}\left\|v_{n}^{(m-1)}-r\right\|\right\}\right. \\
& +L^{2} b_{n}^{(m)} b_{n}^{(m-1)} \sup _{n \geq 0}\left\{c_{n}^{(m-2)}\left\|v_{n}^{(m-2)}-r\right\|\right\}
\end{aligned}
$$




$$
\begin{aligned}
& +L^{3} b_{n}^{(m)} b_{n}^{(m-1)} b_{n}^{(m-2)} \sup _{n \geq 0}\left\{c_{n}^{(m-3)}\left\|v_{n}^{(m-3)}-r\right\|\right\} \\
& +\cdots+L^{m-2} b_{n}^{(m)} b_{n}^{(m-1)} b_{n}^{(m-2)} \cdots b_{n}^{(3)} \sup _{n \geq 0}\left\{c_{n}^{(2)}\left\|v_{n}^{(2)}-r\right\|\right\} \\
& \left.+L^{m-1} b_{n}^{(m)} b_{n}^{(m-1)} b_{n}^{(m-2)} b_{n}^{(m-3)} \cdots b_{n}^{(2)} \sup _{n \geq 0}\left\{c_{n}^{(1)}\left\|v_{n}^{(1)}-r\right\|\right\}\right] \\
& =(1-\theta h)\left\|u_{n}-r\right\|+D, \quad n \geq 0 .
\end{aligned}
$$

Now, putting

$$
\begin{aligned}
\alpha_{n}= & \left\|u_{n}-r\right\|, \quad \eta=1-\theta h \quad \text { and } \\
\beta_{n}= & \lambda^{-1}(1+L)\left[\sup _{n \geq 0}\left\{c_{n}^{(m)}\left\|v_{n}^{(m)}-r^{*}\right\|\right\}+L b_{n}^{(m)} \sup _{n \geq 0}\left\{c_{n}^{(m-1)}\left\|v_{n}^{(m-1)}-r^{*}\right\|\right\}\right. \\
& +L^{2} b_{n}^{(m)} b_{n}^{(m-1)} \sup _{n \geq 0}\left\{c_{n}^{(m-2)}\left\|v_{n}^{(m-2)}-r^{*}\right\|\right\} \\
& +L^{3} b_{n}^{(m)} b_{n}^{(m-1)} b_{n}^{(m-2)} \sup _{n \geq 0}\left\{c_{n}^{(m-3)}\left\|v_{n}^{(m-3)}-r^{*}\right\|\right\} \\
& +\cdots+L^{m-2} b_{n}^{(m)} b_{n}^{(m-1)} b_{n}^{(m-2)} \cdots b_{n}^{(3)} \sup _{n \geq 0}\left\{c_{n}^{(2)}\left\|v_{n}^{(2)}-r^{*}\right\|\right\} \\
& \left.+L^{m-1} b_{n}^{(m)} b_{n}^{(m-1)} b_{n}^{(m-2)} b_{n}^{(m-3)} \cdots b_{n}^{(2)} \sup _{n \geq 0}\left\{c_{n}^{(1)}\left\|v_{n}^{(1)}-r^{*}\right\|\right\}\right], \quad \forall n \geq 0,
\end{aligned}
$$

we get $0 \leq \eta<1$ and $\lim _{n \rightarrow \infty} \beta_{n}=0$. Hence, by an application of Lemma 2.5 , we obtain

$$
\lim _{n \rightarrow \infty}\left\|u_{n}-r\right\|=0
$$

This ensures that the sequence $\left\{u_{n}\right\}_{n=0}^{\infty}$ converges strongly to the common fixed $r$ of $T$ and $S$.

(ii) Again, from (3.27), we find

$$
\begin{aligned}
\| w_{n+1} & -r \| \\
\leq & \left(1-\theta b_{n}^{(m)}\right)\left\|w_{n}-r\right\|+\lambda^{-1}(1+L)\left[c_{n}^{(m)}\left\|v_{n}^{(m)}-r\right\|+L\left[b_{n}^{(m)} c_{n}^{(m-1)}\left\|v_{n}^{(m-1)}-r\right\|\right.\right. \\
& +L\left[b_{n}^{(m)} b_{n}^{(m-1)} c_{n}^{(m-2)}\left\|v_{n}^{(m-2)}-r\right\|+L\left[b_{n}^{(m)} b_{n}^{(m-1)} b_{n}^{(m-2)} c_{n}^{(m-3)}\left\|v_{n}^{(m-3)}-r\right\|\right.\right. \\
& +\cdots+L\left[b_{n}^{(m)} b_{n}^{(m-1)} b_{n}^{(m-2)} \cdots b_{n}^{(3)} c_{n}^{(2)}\left\|v_{n}^{(2)}-r\right\|\right. \\
& \left.\left.\left.\left.\left.+L\left[b_{n}^{(m)} b_{n}^{(m-1)} b_{n}^{(m-2)} b_{n}^{(m-3)} \cdots b_{n}^{(2)} c_{n}^{(1)}\left\|v_{n}^{(1)}-r\right\|\right]\right]\right]\right] \cdots\right]\right]+\mu_{n} \\
\leq & (1-\theta h)\left\|w_{n}-r\right\|+\lambda^{-1}(1+L)\left[c_{n}^{(m)}\left\|v_{n}^{(m)}-r\right\|+L\left[c_{n}^{(m-1)}\left\|v_{n}^{(m-1)}-r\right\|\right.\right. \\
& +L\left[c_{n}^{(m-2)}\left\|v_{n}^{(m-2)}-r\right\|+L\left[c_{n}^{(m-3)}\left\|v_{n}^{(m-3)}-r\right\|\right.\right. \\
& \left.\left.\left.\left.+\cdots+L\left[c_{n}^{(2)}\left\|v_{n}^{(2)}-r\right\|+L\left[c_{n}^{(1)}\left\|v_{n}^{(1)}-r\right\|\right]\right]\right]\right] \cdots\right]\right]+\mu_{n} .
\end{aligned}
$$

(iii) Considering $\lim _{n \rightarrow \infty} w_{n}=r$, we have $\lim _{n \rightarrow \infty} w_{n+1}=r$.

Now, using (3.27), we get

$$
\mu_{n}=\left\|w_{n+1}-x_{n}\right\|
$$




$$
\begin{aligned}
\leq & \left\|w_{n+1}-r\right\|+\left\|x_{n}-r\right\| \\
\leq & \left\|w_{n+1}-r\right\|+(1-\theta h)\left\|w_{n}-r\right\| \\
& +\lambda^{-1}(1+L) b_{n}^{(m)}\left[\delta_{n}\left\|v_{n}^{(m)}-r\right\|+L\left[c_{n}^{(m-1)}\left\|v_{n}^{(m-1)}-r\right\|\right.\right. \\
& +L\left[b_{n}^{(m-1)} c_{n}^{(m-2)}\left\|v_{n}^{(m-2)}-r\right\|+L\left[b_{n}^{(m-1)} b_{n}^{(m-2)} c_{n}^{(m-3)}\left\|v_{n}^{(m-3)}-r\right\|\right.\right. \\
& +\cdots+L\left[b_{n}^{(m-1)} b_{n}^{(m-2)} \cdots b_{n}^{(3)} c_{n}^{(2)}\left\|v_{n}^{(2)}-r\right\|\right. \\
& \left.\left.\left.\left.\left.+L\left[b_{n}^{(m-1)} b_{n}^{(m-2)} b_{n}^{(m-3)} \cdots b_{n}^{(2)} c_{n}^{(1)}\left\|v_{n}^{(1)}-r\right\|\right]\right]\right]\right] \cdots\right]\right] \rightarrow 0,
\end{aligned}
$$

as $n \rightarrow \infty$, this means that $\lim _{n \rightarrow \infty} \mu_{n}=0$.

Conversely, suppose that $\lim _{n \rightarrow \infty} \mu_{n}=0$. Now, by setting

$$
\begin{aligned}
\alpha_{n}= & \left\|w_{n}-r\right\|, \quad \eta=1-\theta h \quad \text { and } \\
\beta_{n}= & \lambda^{-1}(1+L)\left[\sup _{n \geq 0}\left\{c_{n}^{(m)}\left\|v_{n}^{(m)}-r^{*}\right\|\right\}+L b_{n}^{(m)} \sup _{n \geq 0}\left\{c_{n}^{(m-1)}\left\|v_{n}^{(m-1)}-r^{*}\right\|\right\}\right. \\
& +L^{2} b_{n}^{(m)} b_{n}^{(m-1)} \sup _{n \geq 0}\left\{c_{n}^{(m-2)}\left\|v_{n}^{(m-2)}-r^{*}\right\|\right\} \\
& +L^{3} b_{n}^{(m)} b_{n}^{(m-1)} b_{n}^{(m-2)} \sup _{n \geq 0}\left\{c_{n}^{(m-3)}\left\|v_{n}^{(m-3)}-r^{*}\right\|\right\} \\
& +\cdots+L^{m-2} b_{n}^{(m)} b_{n}^{(m-1)} b_{n}^{(m-2)} \cdots b_{n}^{(3)} \sup _{n \geq 0}\left\{c_{n}^{(2)}\left\|v_{n}^{(2)}-r^{*}\right\|\right\} \\
& \left.+L^{m-1} b_{n}^{(m)} b_{n}^{(m-1)} b_{n}^{(m-2)} b_{n}^{(m-3)} \cdots b_{n}^{(2)} \sup _{n \geq 0}\left\{c_{n}^{(1)}\left\|v_{n}^{(1)}-r^{*}\right\|\right\}\right]+\mu_{n}, \quad \forall n \geq 0,
\end{aligned}
$$

we get $0 \leq \eta<1$ and $\lim _{n \rightarrow \infty} \beta_{n}=0$. Hence, by an application of Lemma 2.5 , we obtain

$$
\lim _{n \rightarrow \infty}\left\|w_{n}-r\right\|=0
$$

This completes the proof.

The following corollaries show the convergence, almost common-stability and commonstability for the corresponding modified multi-step Noor iterative procedure without errors for two Lipschitz strictly hemicontractive-type mappings in arbitrary Banach spaces.

Corollary 3.4 Let B be a nonempty closed convex subset of $X$ and $T$ and $S$ be two Lipschitz strictly hemicontractive-type mappings from $B$ into itself. Suppose that $\left\{\alpha_{n}^{(i)}\right\}_{n=0}^{\infty},\left\{\beta_{n}^{(i)}\right\}_{n=0}^{\infty}$ for each $i \in\{1,2, \ldots, m\}$ are any appropriate real sequences in $[0,1]$ satisfying the following conditions:

(6) $\alpha_{n}^{(i)}+\beta_{n}^{(i)}=1$, for each $i \in\{1,2,3, \ldots, m\}$,

(7) $\sum_{n=0}^{\infty} \alpha_{n}^{(j)}=\infty$ for each $j \in\{2,3, \ldots, m\}$,

(8)

$$
\begin{aligned}
& L\left[(1+L) \beta_{n}^{(m)}+(1+L)^{2}\left[\beta_{n}^{(m-1)}+L(1+L)^{2} \beta_{n}^{(m-1)} \beta_{n}^{(m-2)}+L^{2} \beta_{n}^{(m-1)} \beta_{n}^{(m-2)} \beta_{n}^{(m-3)}\right.\right. \\
& \left.\left.\quad+\cdots+L^{m-3} \beta_{n}^{(m-1)} \beta_{n}^{(m-2)} \cdots \beta_{n}^{(2)}+L^{m-2} \beta_{n}^{(m-1)} \beta_{n}^{(m-2)} \cdots \beta_{n}^{(2)} \beta_{n}^{(1)}\right]\right] \\
& \quad+\lambda(\lambda-\theta), \quad n \geq 0,
\end{aligned}
$$

where $\theta$ is a constant in $(0, \lambda)$ and $\lambda \in(0,1)$. 
For $u_{0} \in B$, we assume an iterative sequence $\left\{u_{n}\right\}_{n=0}^{\infty}$ defined by

$$
\begin{aligned}
& u_{n+1}=u_{n}^{(m)}=\alpha_{n}^{(m)} u_{n}+\beta_{n}^{(m)} T u_{n}^{(m-1)}, \\
& u_{n}^{(m-1)}=\alpha_{n}^{(m-1)} u_{n}+\beta_{n}^{(m-1)} T u_{n}^{(m-2)}, \\
& \ldots \ldots \\
& u_{n}^{(2)}=\alpha_{n}^{(2)} u_{n}+\beta_{n}^{(2)} T u_{n}^{(1)}, \\
& u_{n}^{(1)}=\alpha_{n}^{(1)} u_{n}+\beta_{n}^{(1)} S u_{n}, \quad n \geq 0
\end{aligned}
$$

and let $\left\{w_{n}\right\}_{n=0}^{\infty}$ be any sequence in $B$ and $\left\{\mu_{n}\right\}_{n=0}^{\infty}$ be a sequence defined by

$$
\mu_{n}=\left\|w_{n+1}-x_{n}\right\|, \quad n \geq 0,
$$

where

$$
\begin{aligned}
& x_{n}=x_{n}^{(m)}=\alpha_{n}^{(m)} w_{n}+\beta_{n}^{(m)} T x_{n}^{(m-1)}, \\
& x_{n}^{(m-1)}=\alpha_{n}^{(m-1)} w_{n}+\beta_{n}^{(m-1)} T x_{n}^{(m-2)}, \\
& \cdots \cdots \\
& x_{n}^{(2)}=\alpha_{n}^{(2)} w_{n}+\beta_{n}^{(2)} T x_{n}^{(1)}, \\
& x_{n}^{(1)}=\alpha_{n}^{(1)} w_{n}+\beta_{n}^{(1)} S w_{n}, \quad n \geq 0 .
\end{aligned}
$$

Then

(i) the iterative sequence $\left\{u_{n}\right\}_{n=0}^{\infty}$ given by (3.29) converges strongly to the common fixed $r$ of $T$ and $S$,

(ii) $\sum_{n=0}^{\infty} \mu_{n}<\infty$ implies that $\lim _{n \rightarrow \infty} w_{n}=r$, so that $\left\{u_{n}^{(m)}\right\}_{n=0}^{\infty}$ is almost common-stable on $B$,

(iii) $\lim _{n \rightarrow \infty} w_{n}=r$, implies that $\lim _{n \rightarrow \infty} \mu_{n}=0$.

Proof The proof follows from the proof of Theorem 3.1 and, for brevity, here we omit it.

Corollary 3.5 Let $B, X, T, S,\left\{u_{n}\right\}_{n=0}^{\infty},\left\{x_{n}\right\}_{n=0}^{\infty},\left\{w_{n}\right\}_{n=0}^{\infty}$ and $\left\{\mu_{n}\right\}_{n=0}^{\infty}$ be as in Corollary 3.4 and $\theta$ be as in Theorem 3.1. Suppose that $\left\{\alpha_{n}^{(i)}\right\}_{n=0}^{\infty},\left\{\beta_{n}^{(i)}\right\}_{n=0}^{\infty}$ for each $i \in\{1,2, \ldots, m\}$ are any appropriate real sequences in $[0,1]$ satisfying the similar condition of condition (3) of Theorem 3.1 and the conditions (6), (7) and (8) of Corollary 3.4 along with the following property:

$$
\beta_{n}^{(m)} \geq h>0, \quad \forall n \geq 0,
$$

where $h$ is a constant.

Then

(i) the iterative sequence $\left\{u_{n}\right\}_{n=0}^{\infty}$ given by (3.29) converges strongly to the common fixed $r$ of $T$ and $S$ and the following inequality holds:

$$
\left\|u_{n+1}-r\right\| \leq(1-\theta h)\left\|u_{n}-r\right\|, \quad \forall n \geq 0
$$

(ii) $\left\|w_{n+1}-r\right\| \leq(1-\theta h)\left\|w_{n}-r\right\|+\mu_{n}, \forall n \geq 0$,

(iii) $\lim _{n \rightarrow \infty} w_{n}=r$, if and only if $\lim _{n \rightarrow \infty} \mu_{n}=0$. 
Proof The proof follows from the proof of Theorem 3.3 and, for brevity, here we omit it.

\section{Remark 3.6}

(a) If we put $m=2$ in our Theorem 3.1, Theorem 3.2 and Theorem 3.3, then we can easily establish Theorem 9, Theorem 10 and Theorem 11 of Hussain et al. [1], respectively. Therefore, we can comment that the results of Hussain et al. [1] are special case of our results.

(b) Since the MIP given by Mann [28], the IIP given by Ishikawa [29], the NIP given by Xu and Noor [30], the MIPE given in Liu [31] and Xu [32], the IIPE given by Liu [31] and $\mathrm{Xu}$ [32] and the TIPE given by Cho et al. [33] are all special cases of our newly proposed MMNIPE given by (1.5), by setting the appropriate values of $m$ and $c_{n}^{(m)}$ in our Theorem 3.1, Theorem 3.2 and Theorem 3.3, we can easily obtain the convergence, almost common-stability and common-stability criteria of the above-mentioned iterative procedures for two Lipschitz strictly hemicontractive-type mappings in arbitrary Banach spaces.

\section{Examples}

In this section, we provide a numerical example to verify our analytical results and to show a numerical comparison between our newly proposed MMNIPE given by (1.5) and some other most analogous iterative procedures with errors.

Example 4.1 Consider $B$ is a nonempty subset of an arbitrary Banach space $X$ with the usual norm and let $B=\mathbb{R}$. Suppose that $T$ and $S$ are two self-maps on $B$ which are defined as follows:

$$
T u=\frac{1}{3} \sin ^{2} u, \quad \text { and } \quad S u=\frac{2}{3} u^{2} .
$$

Now, if we let $L=\frac{2}{3}, \sigma=\frac{3}{2}, \theta=\frac{1}{300}$, then it is obvious that $F_{T} \cap F_{S}=\{0\}, \lambda=\frac{\sigma-1}{\sigma}=\frac{3 / 2-1}{3 / 2}=$ $\frac{1}{3} \in(0,1)$ and

$$
\begin{aligned}
& \left|T u_{1}-T u_{2}\right|=\frac{1}{3}\left|\sin ^{2} u_{1}-\sin ^{2} u_{2}\right| \leq \frac{2}{3}\left|\sin u_{1}-\sin u_{2}\right|\left|\sin u_{1}+\sin u_{2}\right| \leq L\left|u_{1}-u_{2}\right|, \\
& \left|S u_{1}-S u_{2}\right|=\frac{2}{3}\left|u_{1}^{2}-u_{2}^{2}\right|=\frac{2}{3}\left|u_{1}-u_{2}\right|\left|u_{1}+u_{2}\right| \leq L\left|u_{1}-u_{2}\right|, \quad \forall u_{1}, u_{2} \in \mathbb{R} .
\end{aligned}
$$

Hence, both $T$ and $S$ are Lipschitzian mappings on $B$.

Likewise, using (1.1) we have

$$
\begin{aligned}
& \left|\left(1+t^{\prime}\right)(q-r)-t^{\prime} t(T q-T r)\right| \\
& \quad \geq\left(1+t^{\prime}\right)|q-r|-t^{\prime} t|T q-T r| \\
& \quad=|q-r|+t^{\prime}(|q-r|-t|T q-T r|) \\
& \quad \geq|q-r|+t^{\prime}(|q-r|-t L|q-r|)=\left(1+t^{\prime}(1-t L)\right)|q-r| \\
& \quad \geq\|q-r\|
\end{aligned}
$$

for any $q, r \in \mathbb{R}$ and $t^{\prime}>0$. Therefore, $T$ is strongly pseudocontractive and hence Lemma 2.8 confirms that $T$ is strictly hemicontractive on $B$. Also, the similar arguments 
hold for the mapping $S$. Hence both $T$ and $S$ are Lipschitz strictly hemicontractive mappings on $B$.

Now, if we consider

$$
\begin{aligned}
& b_{n}^{(m)}=\frac{5}{9} \cdot \frac{1}{\sqrt{n}+100}, \quad c_{n}^{(m)}=\frac{1}{(\sqrt{n}+100)^{2}}, \quad a_{n}^{(m)}=1-\left(b_{n}^{(m)}+c_{n}^{(m)}\right), \\
& b_{n}^{(j)}=c_{n}^{(j)}=\frac{3}{5} \cdot \frac{1}{n+100}, \quad a_{n}^{(j)}=1-\left(b_{n}^{(j)}+c_{n}^{(j)}\right),
\end{aligned}
$$

where $j=1,2, \ldots, m-1$, and $\forall n \geq 0$.

Then, for $n=0, m=10$, we obtain

$$
\begin{aligned}
L[ & (1+L) b_{n}^{(m)}+(1+L)^{2} b_{n}^{(m-1)}+L(1+L)^{2} b_{n}^{(m-1)} b_{n}^{(m-2)} \\
& +L^{2}(1+L)^{2} b_{n}^{(m-1)} b_{n}^{(m-2)} b_{n}^{(m-3)}+\cdots+L^{m-3}(1+L)^{2} b_{n}^{(m-1)} b_{n}^{(m-2)} b_{n}^{(m-3)} \cdots b_{n}^{(2)} \\
& +L^{m-2}(1+L)^{2} b_{n}^{(m-1)} b_{n}^{(m-2)} b_{n}^{(m-3)} \cdots b_{n}^{(2)} b_{n}^{(1)} \\
& +\left[c_{n}^{(m)}+(1+L) c_{n}^{(m-1)}+L(1+L) b_{n}^{(m-1)} c_{n}^{(m-2)}\right. \\
& +L^{2}(1+L) b_{n}^{(m-1)} b_{n}^{(m-2)} c_{n}^{(m-3)}+\cdots+L^{m-3}(1+L) b_{n}^{(m-1)} b_{n}^{(m-2)} \cdots b_{n}^{(3)} c_{n}^{(2)} \\
& \left.\left.+L^{m-2}(1+L) b_{n}^{(m-1)} b_{n}^{(m-2)} b_{n}^{(m-3)} \cdots b_{n}^{(2)} c_{n}^{(1)}\right]\right]+\frac{c_{n}^{(m)}}{b_{n}^{(m)}} \leq 0.042231081 \leq 0.11 .
\end{aligned}
$$

Therefore, by an application of Theorem 3.1, we can say that the iterative sequence $\left\{u_{n}\right\}_{n=0}^{\infty}$ defined by (1.5) converges strongly to the common fixed 0 of $T$ and $S$ in $B$ and the corresponding MMNIPE given by (1.5) is common-stable as well as almost common-stable on $B$.

Analogously, by applying Theorem 3.2 and Theorem 3.3, we can easily prove that the iterative sequence $\left\{u_{n}\right\}_{n=0}^{\infty}$ defined by (1.5) converges strongly to the common fixed 0 of $T$ and $S$ in $B$ and the corresponding MMNIPE given by (1.5) is common-stable as well as almost common-stable on $B$.

For the numerical experiment, here we consider our newly proposed MMNIPE given by (1.5) for $m=10$, and compared it with the STIIPE given by Hussain et al. [1], the IIPE given

Table 1 Numerical results corresponding to $u_{0}=0.5$ for 1000 iterative steps

\begin{tabular}{cllll}
\hline Step no. & Our proposed MMNIPE & STIIPE of Hussain et al. & IIPE of Liu & TIPE of Cho et al. \\
\hline 1 & 0.5 & 0.5 & 0.5 & 0.5 \\
2 & 0.497667276003391 & 0.497668105410462 & 0.998544685005923 & 0.497667277380927 \\
3 & 0.495335888378632 & 0.495337523105695 & 1.328130014700319 & 0.495335891074855 \\
4 & 0.493012550083953 & 0.493014966750906 & 1.572697385396054 & 0.493012554043365 \\
5 & 0.490699600582596 & 0.490702776576019 & 1.765873713917432 & 0.490699605752504 \\
$\ldots$ & $\ldots$ & $\ldots$ & $\ldots$ & $\ldots$ \\
25 & 0.446858824895112 & 0.446873361526477 & 3.033120278084475 & 0.446858846100032 \\
$\ldots$ & $\ldots$ & $\ldots$ & $\ldots$ & $\ldots$ \\
50 & 0.397841478361139 & 0.397863341508779 & 3.297911825793324 & 0.397841507128528 \\
$\ldots$ & $\ldots$ & $\ldots$ & $\ldots$ & $\ldots$ \\
100 & 0.315560781936281 & 0.315586416536455 & 3.153669674069195 & 0.315560811436780 \\
$\ldots$ & $\ldots$ & $\ldots$ & $\ldots$ & $\ldots$ \\
500 & 0.049930372833121 & 0.049936108842272 & 1.278420609396875 & 0.049930378403359 \\
$\ldots$ & $\ldots$ & $\ldots$ & $\ldots$ & $\ldots$ \\
1000 & 0.005520028104577 & 0.005520671212411 & 0.526921124813466 & 0.005520028727839 \\
\hline
\end{tabular}




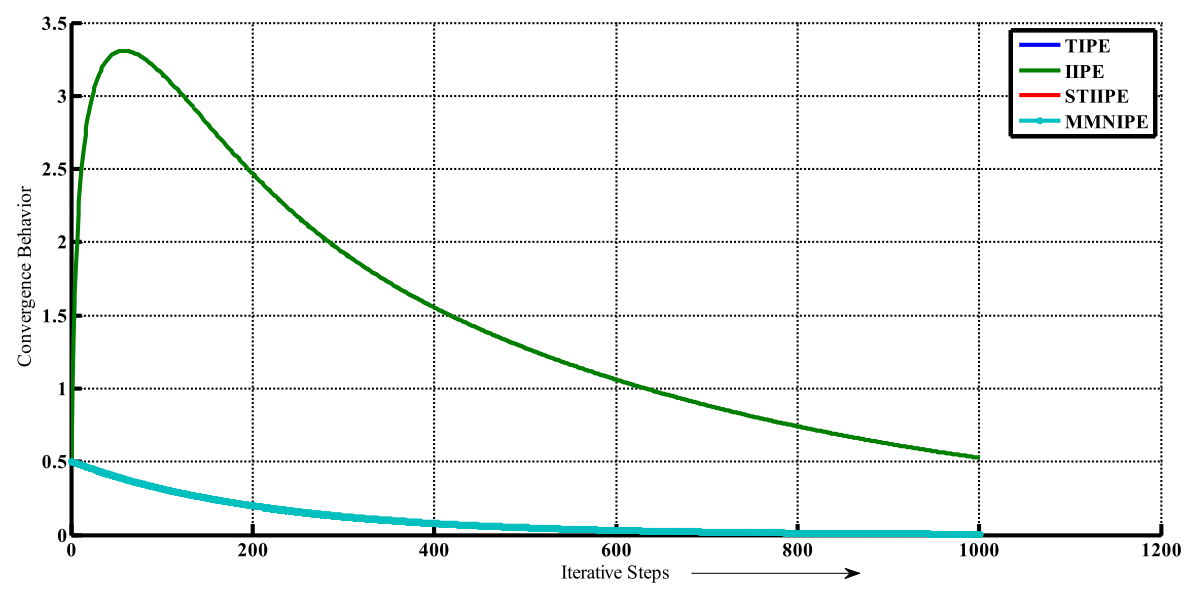

Figure 1 Convergence behavior corresponding to $u_{0}=0.5$ for 1000 iterative steps

by Liu [31], and the TIPE given by Cho et al. [33]. By using MATLAB programming language, we computed the different iterative steps and the numerical comparison is shown in Table 1. Furthermore, the convergence behaviors of these iterative procedures with errors are shown in Fig. 1. For all iterative procedure, we take the initial approximation $u_{0}=0.5$. For our proposed MMNIPE given by (1.5), we consider $v_{n}^{(i)}=\frac{1}{n+1}$, where $i=1,2,3, \ldots, 10$ and

$$
\begin{aligned}
& b_{n}^{(10)}=\frac{5}{9} \cdot \frac{1}{\sqrt{n}+100}, \quad c_{n}^{(10)}=\frac{1}{(\sqrt{n}+100)^{2}}, \\
& a_{n}^{(10)}=1-\left(b_{n}^{(10)}+c_{n}^{(10)}\right)=\frac{9 n+1795 \sqrt{n}+89,491}{9(\sqrt{n}+100)^{2}}, \\
& b_{n}^{(j)}=c_{n}^{(j)}=\frac{3}{5} \cdot \frac{1}{n+100}, \quad a_{n}^{(j)}=1-\left(b_{n}^{(j)}+c_{n}^{(j)}\right)=\frac{5 n+494}{5(n+100)},
\end{aligned}
$$

where $j=1,2,3, \ldots, 9$ and $\forall n \geq 0$.

For the STIIPE given by Hussain et al. [1], we consider $v_{n}^{(i)}=\frac{1}{n+1}$, where $i=1,2$ and

$$
\begin{aligned}
& b_{n}^{(2)}=\frac{5}{9} \cdot \frac{1}{\sqrt{n}+100}, \quad c_{n}^{(2)}=\frac{1}{(\sqrt{n}+100)^{2}}, \\
& a_{n}^{(2)}=1-\left(b_{n}^{(2)}+c_{n}^{(2)}\right)=\frac{9 n+1795 \sqrt{n}+89,491}{9(\sqrt{n}+100)^{2}}, \\
& b_{n}^{(1)}=c_{n}^{(1)}=\frac{3}{5} \cdot \frac{1}{n+100}, \quad a_{n}^{(1)}=1-\left(b_{n}^{(1)}+c_{n}^{(1)}\right)=\frac{5 n+494}{5(n+100)} \quad \text { and } \quad \forall n \geq 0 .
\end{aligned}
$$

For the IIPE given by Liu [31], we consider $v_{n}^{(i)}=\frac{1}{n+1}$, where $i=1,2$ and

$$
\begin{aligned}
& b_{n}^{(2)}=\frac{5}{9} \cdot \frac{1}{\sqrt{n}+100}, \quad a_{n}^{(2)}=1-b_{n}^{(2)}=\frac{9 \sqrt{n}+895}{9(\sqrt{n}+100)}, \\
& b_{n}^{(1)}=\frac{3}{5} \cdot \frac{1}{n+100}, \quad a_{n}^{(1)}=1-b_{n}^{(1)}=\frac{5 n+497}{5(n+100)} \quad \text { and } \quad \forall n \geq 0 .
\end{aligned}
$$


For the TIPE given by Cho et al. [33], we consider $v_{n}^{(i)}=\frac{1}{n+1}$, where $i=1,2,3$ and

$$
\begin{aligned}
& b_{n}^{(3)}=\frac{5}{9} \cdot \frac{1}{\sqrt{n}+100}, \quad c_{n}^{(3)}=\frac{1}{(\sqrt{n}+100)^{2}}, \\
& a_{n}^{(3)}=1-\left(b_{n}^{(3)}+c_{n}^{(3)}\right)=\frac{9 n+1795 \sqrt{n}+89,491}{9(\sqrt{n}+100)^{2}}, \\
& b_{n}^{(j)}=c_{n}^{(j)}=\frac{3}{5} \cdot \frac{1}{n+100}, \quad a_{n}^{(j)}=1-\left(b_{n}^{(j)}+c_{n}^{(j)}\right)=\frac{5 n+494}{5(n+100)},
\end{aligned}
$$

where $j=1,2$ and $\forall n \geq 0$.

The comparison table (Table 1) confirms that the rate of convergence of our proposed MMNIPE given by (1.5) is better than that of the STIIPE given by Hussain et al. [1], the IIPE given by Liu [31] and the TIPE given by Cho et al. [33].

\section{Conclusion}

In this study, we established the convergence, almost common-stability and commonstability criteria of our proposed MMNIPE given by (1.5) for two Lipschitz strictly hemicontractive-type mappings in arbitrary Banach spaces. The obtained results of this paper provided easy and straightforward techniques for proving the convergence, almost common-stability and common-stability criteria of the proposed MMNIPE given by (1.5). Furthermore, the results of this paper extended the corresponding results of Hussain et al. [1, 7-9], Zegeye et al. [2], Meche et al. [3], Chidume and Osilike [4], Chidume [5], Liu et al. [12], Zeng [13], Yu et al. [11], Yang [25], Chidume [36], Deng [37, 38] and Liu [39]. According to the Remark 3.6, our results generalized and unify the corresponding results of Hussain et al. [1], Mann [28], Ishikawa [29], Xu and Noor [30], Liu [31] and Xu [32] and Cho et al. [33] in the case of establishing the fixed-point theorem-based iterative procedures for two Lipschitz strictly hemicontractive-type mappings. At the end of this work, we discussed a computational numerical example which verify our main results and compare the performance of our proposed MMNIPE given by (1.5) with other most analogous iterative procedures with errors. From the comparison table (Table 1), we conclude that our proposed MMNIPE given by (1.5) superior over the STIIPE given by Hussain et al. [1] and the TIPE given by Cho et al. [33] in the case of convergence at the common fixed point of two Lipschitz strictly hemicontractive-type mappings.

\section{Acknowledgements}

We are really grateful to the journal authority for waiving the article processing charges.

\section{Funding}

We have no funding from any source for this article.

Availability of data and materials

Data sharing not applicable to this article as no datasets were generated or analyzed during the current study.

Competing interests

The author declares that he has no competing interests.

Authors' contributions

It is a single author paper. The author read and approved the final version of the manuscript.

\section{Publisher's Note}

Springer Nature remains neutral with regard to jurisdictional claims in published maps and institutional affiliations. 
Received: 24 June 2020 Accepted: 21 February 2021 Published online: 08 March 2021

\section{References}

1. Hussain, N., Rafiq, A., Ciric, L.B.: Stability of the Ishikawa iteration scheme with errors for two strictly hemicontractive operators in Banach spaces. Fixed Point Theory Appl. 2012, 160 (2012)

2. Zegeye, H., Meche, T.H., Sangago, M.G.: Algorithms of common solutions for a fixed point of hemicontractive-type mapping and a generalized equilibrium problem. Int. J. Adv. Math. Sci. 5(1), 20-26 (2017)

3. Meche, T.H., Sangago, M.G., Zegeye, H.: Iterative methods for a fixed point of hemicontractive-type mapping and a solution of a variational inequality problem. Creative Math. Inform. 25(2), 175-188 (2016)

4. Chidume, C.E., Osilike, M.O.: Fixed point iterations for strictly hemicontractive maps in uniformly smooth Banach spaces. Numer. Funct. Anal. Optim. 15, 779-790 (1994)

5. Chidume, C.E.: An iterative process for nonlinear Lipschitzian strongly accretive mappings in $L_{p}$ spaces. J. Math. Anal. Appl. 151, 453-461 (1990)

6. Isiogugu, F.O., Osilike, M.O.: Convergence theorems for new classes of multivalued hemicontractive-type mappings. Fixed Point Theory Appl. 2014, 93 (2014)

7. Hussain, N., Sahu, D., Rafiq, A.: Iteration scheme for common fixed points of hemicontractive and nonexpansive operators in Banach spaces. Fixed Point Theory Appl. 2013, 247 (2013)

8. Hussain, N., Ćirić, L.B., Cho, Y.J., Rafiq, A.: On Mann-type iteration method for a family of hemicontractive mappings in Hilbert spaces. J. Inequal. Appl. 2013, 41 (2013)

9. Hussain, N., Rafiq, A., Ciric, L.B., Al-Mezel, S.: Almost stability of the Mann type iteration method with error term involving strictly hemicontractive mappings in smooth Banach spaces. J. Inequal. Appl. 2012, 207 (2012)

10. Olaleru, J., Okeke, G.: Convergence theorems on asymptotically demicontractive and hemicontractive mappings in the intermediate sense. Fixed Point Theory Appl. 2013, 352 (2013)

11. Yu, Y., Wu, Z., Yang, P.X.: An iterative algorithm for hemicontractive mappings in Banach spaces. Abstr. Appl. Anal. 2012, Article ID 264103 (2012)

12. Liu, Z., Kang, S.M., Shim, S.H.: Almost stability of the Mann iteration method with errors for strictly hemicontractive operators in smooth Banach spaces. J. Korean Math. Soc. 40(1), 29-40 (2003)

13. Zeng, L.C.: Iterative approximation of solutions to nonlinear equations of strongly accretive operators in Banach spaces. Nonlinear Anal. TMA 31, 589-598 (1998)

14. Deng, L., Ding, X.P.: Iterative approximation of Lipschitz strictly pseudocontractive mappings in uniformly smooth Banach spaces. Nonlinear Anal., Theory Methods Appl. 24(7), 981-987 (1995)

15. Deng, L: On Chidume's open questions. J. Math. Anal. Appl. 174(2), 441-449 (1993)

16. Ugwunnadi, G.C., Izuchukwu, C., Mewomo, O.T.: Convergence theorems for generalized hemicontractive mapping in p-uniformly convex metric space. Appl. Anal. 26(2), 221-229 (2020)

17. Agwu, I.K., Igbokwe, D.I.: Hybrid-type iteration scheme for approximating fixed points of Lipschitz $\alpha$-hemicontractive mappings. Adv. Fixed Point Theory 10, 3 (2020)

18. Meche, T.H., Zegeye, H.: On fixed point results for hemicontractive-type multi-valued mapping, finite families of split equilibrium and variational inequality problems. Sahand Commun. Math. Anal. 17(3), 189-217 (2020)

19. Zegeye, H., Tufa, A.R.: Halpern-Ishikawa type iterative method for approximating fixed points of non-self pseudocontractive mappings. Fixed Point Theory Appl. 2018, 15 (2018)

20. Isiogugu, F.O.: On approximation of fixed points of multivalued pseudocontractive mappings in Hilbert spaces. Fixed Point Theory Appl. 2016, 59 (2016)

21. Dashputre, S., Das, A.K.: On convergence theorems for new classes of multivalued hemicontractive-type mappings. Glob. J. Pure Appl. Math. 12(1), 105-116 (2016)

22. Kim, G.E., Kim, T.H.: Mann and Ishikawa iterations with errors for non-Lipschitzian mappings in Banach spaces. Comput. Math. Appl. 42, 1565-1570 (2001)

23. Kim, T.H.: Approximation of common fixed points for a family of non-Lipschitzian self-mappings. Nonlinear Anal. Convex Anal. 2001, 1(1187), 165-175 (2001)

24. Plubtieng, S., Wangkeeree, R.: Strong convergence theorem for multi-step Noor iterations with errors in Banach spaces. J. Math. Anal. Appl. 321, 10-23 (2006)

25. Yang, L., Peng, S.: Convergence and stability of modified Ishikawa iteration sequence with errors. Fixed Point Theory Appl. 2014, 224 (2014)

26. Thianwan, T:: Convergence criteria of modified Noor iterations with errors for three asymptotically nonexpansive nonself-mappings. J. Nonlinear Sci. Appl. 6, 181-197 (2013)

27. Zeng, L.C., Yao, J.C.: Stability of iterative procedures with errors for approximating common fixed points of a couple of q-contractive-like mappings in Banach spaces. J. Math. Anal. Appl. 321, 661-674 (2006)

28. Mann, W.R.: Mean value methods in iteration. Proc. Am. Math. Soc. 4, 506-510 (1953)

29. Ishikawa, S.: Fixed point by a new iteration. Proc. Am. Math. Soc. 44, 147-150 (1974)

30. Xu, B.L., Noor, M.A.: Fixed-point iterations for asymptotically nonexpansive mappings in Banach spaces. J. Math. Anal. Appl. 267, 444-453 (2002)

31. Liu, L.S.: Ishikawa and Mann iterative processes with errors for nonlinear strongly accretive mappings in Banach spaces. J. Math. Anal. Appl. 194, 114-125 (1995)

32. $\mathrm{Xu}, \mathrm{Y}$ : Ishikawa and Mann iterative processes with errors for nonlinear strongly accretive operator equations. J. Math. Anal. Appl. 224, 91-101 (1998)

33. Cho, Y.J., Zhou, H., Guo, G.: Weak and strong convergence theorems for three-step iterations with errors for asymptotically nonexpansive mappings. Comput. Math. Appl. 47, 707-717 (2004)

34. Weng, X.: Fixed point iteration for local strictly pseudo-contractive mapping. Proc. Am. Math. Soc. 113(3), 727-731 (1991)

35. Kato, T.: Nonlinear semigroups and evolution equations. J. Math. Soc. Jpn. 19, 508-520 (1967)

36. Chidume, C.E.: Iterative solution of nonlinear equations in smooth Banach spaces. Nonlinear Anal. TMA 26(11), 1823-1834 (1996)

37. Deng, L:: An iterative process for nonlinear Lipschitz and strongly accretive mappings in uniformly convex and uniformly smooth Banach spaces. Acta Appl. Math. 32, 183-196 (1993) 
38. Deng, L.: Iteration processes for nonlinear Lipschitz strongly accretive mappings in $L_{p}$ spaces. J. Math. Anal. Appl. 188(1), 128-140 (1994)

39. Liu, L.S.: Ishikawa and Mann iteration process with errors for nonlinear strongly accretive mappings in Banach spaces. J. Math. Anal. Appl. 194(1), 114-125 (1995)

40. Berinde, V:: Generalized Contractions and Applications. Editura Cub Press 22, Baia Mare (1997) (Romanian)

Submit your manuscript to a SpringerOpen ${ }^{\circ}$ journal and benefit from:

- Convenient online submission

$\checkmark$ Rigorous peer review

- Open access: articles freely available online

- High visibility within the field

- Retaining the copyright to your article

Submit your next manuscript at $\gg$ springeropen.com 